\title{
Feedforward Control for Underactuated Bipedal Walking on Varying Compliant Slopes
}

\begin{tabular}{|c|c|}
\hline Journal: & Transactions of the Canadian Society for Mechanical Engineering \\
\hline Manuscript ID & TCSME-2017-0020.R2 \\
\hline Manuscript Type: & Article \\
\hline Date Submitted by the Author: & 30-Nov-2017 \\
\hline Complete List of Authors: & $\begin{array}{l}\text { Yao, Daojin; Wuhan University, School of Power and Mechanical } \\
\text { Engineering } \\
\text { He, Siyu; Wuhan University, School of Power and Mechanical Engineering } \\
\text { Wu, Yao; Wuhan University, School of Power and Mechanical Engineering } \\
\text { Xiao, Xiaohui; Wuhan University, School of Power and Mechanical } \\
\text { Engineering } \\
\text { Wang, Yang; Wuhan University, School of Power and Mechanical } \\
\text { Engineering }\end{array}$ \\
\hline \multirow[t]{2}{*}{$\begin{array}{r}\text { Is the invited manuscript for } \\
\text { consideration in a Special } \\
\text { Issue? : }\end{array}$} & N/A \\
\hline & $\begin{array}{l}\text { feedforward control, underactuated walking, biped robot, varying slopes, } \\
\text { compliant ground }\end{array}$ \\
\hline
\end{tabular}




\title{
Feedforward Control for Underactuated Bipedal Walking on Varying Compliant Slopes
}

\author{
Daojin Yao \\ School of Power and Mechanical Engineering \\ Wuhan University, Wuhan, 430072, China \\ E-mail: ydaojin@whu.edu.cn

\section{Siyu He} \\ School of Power and Mechanical Engineering \\ Wuhan University, Wuhan, 430072, China \\ E-mail: syhewhu@whu.edu.cn

\section{Yao Wu} \\ School of Power and Mechanical Engineering \\ Wuhan University, Wuhan, 430072, China \\ E-mail:wuyao@whu.edu.cn

\section{Xiaohui Xiao* (Corresponding Author)} \\ School of Power and Mechanical Engineering \\ Wuhan University, Wuhan, 430072, China \\ E-mail: xhxiao@whu.edu.cn

\section{Yang Wang} \\ School of Power and Mechanical Engineering \\ Wuhan University, Wuhan, 430072, China \\ E-mail: wangyang.hf1987@whu.edu.cn
}




\begin{abstract}
In this paper, a feedforward control strategy is proposed to enable stable underactuated bipedal walking on varying compliant slopes with a known inclination angle to handle with the variation in natural environments. First, spring-damper units were employed in the horizontal and vertical directions to model the compliant ground, which is described as a rigid kinematical chain coupled with a spring-damper system. Second, a new definition of stable underactuated bipedal walking based on the walking speed was provided. Stable walking is achieved by adjusting the velocity of the biped's center of mass $(\mathrm{CoM})$ within limits that have been proved to allow at least one walking cycle. Based on the definition of stability and inspired by the gait characteristics of human walking on varying slopes, the proposed feedforward control strategy was based on the motion state of a robot's CoM. Speed control is realized by adjusting the displacement of the CoM with the change of slope to achieve stable walking. Finally, simulation experiments were conducted to validate the proposed controller. The simulation results demonstrate that stable walking is achieved on varying compliant slopes by implementing the proposed control strategy.
\end{abstract}

Keywords: feedforward control; underactuated walking; biped robot; varying slopes; complaint ground 


\section{Introduction}

Bipedal walking has attracted considerable attention in recent years because its adaptability to a complex environment and has a considerable impact on human-machine interaction (Chen and Huang, 2012). However, a high energy consumption by bipedal robots restricts their development and practical application. Hence, underactuated bipedal walking, a more efficient and simple walking model, has been widely researched in recent years (Collins et al., 2005; Grizzle et al., 2014). Underactuated bipedal walking employs fewer actuators than the degrees of freedom during robot locomotion (Westervelt et al., 2007), resulting in the difficulty in achieving stable walking.

Several control methods have been proposed to stabilize underactuated bipedal walking. Based on hybrid zero dynamics (Westervelt et al., 2003; Wang et al., 2014), planar three-link and five-link biped robots with a torso were studied, and stable walking was realized on level ground by using virtual constraints and feedback linearization. In (Shih et al., 2012), a time-scaling control method was introduced to track the periodic reference trajectory of a planar underactuated bipedal robot. A model predictive control method was used to realize online speed regulation, and dynamic stable walking was achieved (Tao, 2014). Stable walking of a robot on level ground was realized by using these methods, and these methods are based on the hypotheses of a rigid contact model and an instantaneous double support phase. However, in real environments, the ground is compliant and those methods could not be used.

The effect of ground compliance on underactuated bipedal walking has been demonstrated by many researchers (Plestan et al., 2003; Sreenath et al., 2011; Chu et al., 2016). An experiment using RABBIT demonstrated that an additional loss of energy during compliant impact caused a lower speed on the compliant surface. Through experiments with MABEL, Sreenath suggested that a robot's actual walking speed is faster than the simulation speed. In addition to compliant ground, a robot should manage to walk stably in many other human locomotion environments such as slopes, stairs, and uneven terrain (Park et al., 2013). Walking ability of a bipedal robot in these complex 
environments is important for practical applications of the robot.

For a complex environment like varying slopes, reinforcement learning was applied to the controller design of an underactuated robot (Tedrake et al., 2004; Hitomi et al., 2006). Adjusting the parameters online enables the robot to adapt to various types of terrain and slopes in a few minutes. An algorithm that utilizes potential energy shaping control was designed on the basis of the gravity compensation method, which enables a robot to walk stably on various inclined surfaces (Spong and Bullo, 2005). Moreover, energy tracking control was incorporated into the controller design to enlarge the basin of attraction and increase system robustness (Spong et al., 2007). A human-inspired control strategy was employed using AMBER to enable a robot to walk stably in three types of environments: upward sloping ground, downward sloping ground, and flat ground (Yadukumar et al., 2012). Although bipedal walking on varying slopes was realized using these control strategies, the implementation of such walking in practical applications requires the collection of large preprocessing sample data or complex calculations.

The primary contribution of this study lies in the proposed feedforward control strategy for stabilizing underactuated bipedal walking on varying compliant slopes. According to the definition of stable walking "the biped does not fall", a new mathematical definition of underactuated stable walking is provided. In addition to human gait characteristics on varying slopes and the definition of stable walking, the proposed feedforward control strategy is based on the motion state of the center of mass $(\mathrm{CoM})$. The horizontal velocity of a robot's CoM is considered to be the system output. Stable walking can be realized by adjusting the displacement of the CoM within a single walking cycle.

This paper is organized as follows. Section 2 describes a hybrid dynamic model that realizes underactuated bipedal walking on a compliant slope. Section 3 presents a new definition of stable bipedal walking and a feedforward control strategy to stabilize underactuated walking on varying slopes. The proposed control strategy is verified through simulation in Section 4, and conclusions are 
given in Section 5.

\section{Modelling for Bipedal Walking on a Compliant Slope}

This section briefly introduces the dynamics of bipedal locomotion on a compliant slope. The compliant ground is modeled as spring-damper equivalent system (see Fig.1) (Wang et al., 2017; Chemori et al., 2010). The compliant structure contains discrete elastic springs, viscous damp cell arrays, a rigid ground foundation, and surface fragments. The stiffness of the elastic spring is denoted by $k$, and the damping of the viscous damper is denoted by $c$. Each surface fragment is connected to the rigid ground foundation through a pair of spring-damper units in the horizontal and vertical directions. The parameter $\gamma$ is the slope angle of the ground; it is negative for walking downhill and positive for walking uphill. Here, $\gamma$ varies in the range of $\left[-10^{\circ}, 10^{\circ}\right]$.

The biped considered in this paper consists of four rigid links and unactuated point feet (see Fig. 1). The robot has three actuated joints, a hip $\left(q_{3}\right)$ and two knees $\left(q_{2}, q_{4}\right)$. The length of the two thighs and two calves is $l$. The mass of the two thighs and two calves is $m$. The masses of the hip and knee are $m_{\mathrm{h}}$ and $m_{\mathrm{k}}$, respectively. The three joints are controlled by three torques $\left(u_{1}, u_{2}\right.$, $u_{3}$ ). On the basis of Routhian reduction, the hip joint and the foot-ground contact point (CP) are connected using a virtual rod. The underactuated freedom $q_{1}$ is defined as the angle between the virtual rod and slope vertical. Moreover, $q_{1}$ is positive on the right side of the slope vertical, and negative on the left side; $q_{2}, q_{3}$, and $q_{4}$ are positive in the counterclockwise direction. Here, $x_{\mathrm{st}}$ and $y_{\mathrm{st}}$, and $x_{\mathrm{sw}}$ and $y_{\mathrm{sw}}$ are the level and vertical displacement of the support foot and swing foot, respectively.

A step comprises two phases, the single support phase (SSP) and double support phase (DSP). In the SSP, only one leg is in contact with the ground, and in the DSP, both legs are in contact with the ground. Due to the compliance of the ground, the DSP is not instantaneous. During the SSP, the 
robot-ground system is modeled as a rigid planar kinematic chain in one-point contact with a stiffness-damping system. By using the method of Lagrange, the equation of motion is formulated as

$$
\boldsymbol{D}(\boldsymbol{q}) \ddot{\boldsymbol{q}}+\boldsymbol{H}(\boldsymbol{q}, \dot{\boldsymbol{q}})=\boldsymbol{B}_{1} \boldsymbol{F}_{\mathrm{st}}(\boldsymbol{q}, \dot{\boldsymbol{q}})+\boldsymbol{B}_{2} \boldsymbol{u}
$$

where $\boldsymbol{q}=\left[q_{1}, q_{2}, q_{3}, q_{4}, x_{\mathrm{st}}, y_{\mathrm{st}}\right]^{\prime}$ is the structural description and definition of the generalized coordinates, $\dot{\boldsymbol{q}}$ is the velocity of the robot, and $\ddot{\boldsymbol{q}}$ is the robot's accelerated velocity. The matrix $\boldsymbol{D}(\boldsymbol{q})$ is a $(6 \times 6)$ mass-inertia matrix, $\boldsymbol{H}(\boldsymbol{q}, \dot{\boldsymbol{q}})$ is the vector $(6 \times 1)$ of Coriolis and gravity terms, $\boldsymbol{B}_{1}$ is the input matrix $(6 \times 2)$ for the force of the stance leg, $\boldsymbol{F}_{\mathrm{st}}(\boldsymbol{q}, \dot{\boldsymbol{q}})$ is the $(2 \times 1)$ force of the stance leg, $\boldsymbol{B}_{2}$ is the input matrix $(6 \times 6)$ for the actuated joints, and $\boldsymbol{u}$ is the vector $(6 \times 1)$ of input torques. During this phase, $q_{1}, x_{\mathrm{st}}$, and $y_{\mathrm{st}}$ are underactuated. By defining $\boldsymbol{x}:=(\boldsymbol{q} ; \dot{\boldsymbol{q}})$, the model is written in the state-space form as

$$
\begin{aligned}
\dot{\boldsymbol{x}}=\frac{d}{d t}\left[\begin{array}{c}
\boldsymbol{q} \\
\dot{\boldsymbol{q}}
\end{array}\right] & =\left[\begin{array}{c}
\dot{\boldsymbol{q}} \\
\boldsymbol{M}^{-1}(\boldsymbol{q})\left[-\boldsymbol{H}(\boldsymbol{q}, \dot{\boldsymbol{q}})+\boldsymbol{B}_{1} \boldsymbol{F}_{\mathrm{st}}(\boldsymbol{q}, \dot{\boldsymbol{q}})+\boldsymbol{B}_{2} \boldsymbol{u}\right]
\end{array}\right] \\
= & h(\boldsymbol{x})+f_{\mathrm{st}}(\boldsymbol{x})+b(\boldsymbol{x}) \boldsymbol{u}
\end{aligned}
$$

During the DSP, two contact forces are exerted on both legs. By using the method of Lagrange, the equation of motion is formulated as

$$
\boldsymbol{D}(\boldsymbol{q}) \ddot{\boldsymbol{q}}+\boldsymbol{H}(\boldsymbol{q}, \dot{\boldsymbol{q}})=\boldsymbol{B}_{1} \boldsymbol{F}_{\mathrm{st}}(\boldsymbol{q}, \dot{\boldsymbol{q}})+\boldsymbol{E}_{\mathrm{sw}}(\boldsymbol{q})^{\prime} \boldsymbol{F}_{\mathrm{sw}}(\boldsymbol{q}, \dot{\boldsymbol{q}})+\boldsymbol{B}_{2} \boldsymbol{u}
$$

where $\boldsymbol{E}_{\mathrm{sw}}(\boldsymbol{q})$ is a Jacobian matrix $(2 \times 6)$, and $\boldsymbol{F}_{\mathrm{sw}}(\boldsymbol{q}, \dot{\boldsymbol{q}})$ is the $(2 \times 1)$ force of the swing leg. The model is written in the state-space form as

$$
\begin{aligned}
\dot{\boldsymbol{x}} & =\frac{d}{d t}\left[\begin{array}{l}
\boldsymbol{q} \\
\dot{\boldsymbol{q}}
\end{array}\right] \\
& =\left[\begin{array}{c}
\dot{\boldsymbol{q}} \\
\boldsymbol{M}^{-1}(\boldsymbol{q})\left[-\boldsymbol{H}(\boldsymbol{q}, \dot{\boldsymbol{q}})+\boldsymbol{B}_{1} \boldsymbol{F}_{\mathrm{st}}(\boldsymbol{q}, \dot{\boldsymbol{q}})+\boldsymbol{B}_{2} \boldsymbol{u}+\boldsymbol{E}_{\mathrm{sw}}(\boldsymbol{q}) \boldsymbol{F}_{\mathrm{sw}}(\boldsymbol{q}, \dot{\boldsymbol{q}})\right]
\end{array}\right] \\
& =: h(\boldsymbol{x})+f_{\mathrm{st}}(\boldsymbol{x})+b(\boldsymbol{x}) \boldsymbol{u}+f_{\mathrm{sw}}(\boldsymbol{x})
\end{aligned}
$$

The impact map is applied whenever the state enters the switching set $S$ (during the DSP),

$$
S:=\left\{(\boldsymbol{q}, \dot{\boldsymbol{q}}) \mid y_{\mathrm{sw}} \leq 0\right\}
$$


The complete hybrid system can be written as

$$
\begin{cases}\dot{\boldsymbol{x}}=h(\boldsymbol{x})+f_{\mathrm{st}}(\boldsymbol{x})+b(\boldsymbol{x}) \boldsymbol{u} & \boldsymbol{x} \notin S \\ \dot{\boldsymbol{x}}=h(\boldsymbol{x})+f_{\mathrm{st}}(\boldsymbol{x})+b(\boldsymbol{x}) \boldsymbol{u}+f_{\mathrm{sw}}(\boldsymbol{x}) & \boldsymbol{x} \in S\end{cases}
$$

\section{Feedforward Controller Design for Varying Compliant Slopes}

\subsection{Definition of Underactuated Bipedal Stable Walking}

This section provides a sequence of definitions that forms a new definition of underactuated bipedal stable walking. Many researchers have considered walking to be a periodic motion that is represented by periodic orbits in the state space. Moreover, the stability of underactuated walking is determined by analyzing the stability of the periodic orbits (Grizzle et al., 2001). However, bipedal walking does not necessarily have periodic motion. Aperiodic motions such as accelerating, slowing down, and changing direction are essential elements of normal walking. Thus, a general biped walking stability analysis method is required.

The most intuitive definition of stable biped walking is likely that "the biped does not fall" (Yang et al., 2009; Hobbelen and Wisse, 2007). The definition is very simple and intuitive, but it is difficult to rigorously verify the stability of a given controller according to the definition. A new definition of underactuated bipedal stable walking is proposed as follows: if the robot walking speed can always converge to a certain range that have been proved to allow at least one walking cycle, the robot walking is stable.

Definition 1 (Fall). Let $\boldsymbol{Q}_{\text {Fall }}$ be the state space in which the robot walking speed is beyond the range of stable walking speed during a walking cycle. If a robot state $\boldsymbol{x} \in \boldsymbol{Q}_{\text {Fall }}$, robot falls down.

Based on Definition 1, falling is the state in which the robot enters the fall state set. A biped robot is stable if and only if it can always control itself and avoid entering the fall state set. The fall state set $\boldsymbol{Q}_{\text {Fall }}$ can be represented as

$$
\boldsymbol{Q}_{F a l l}=\left\{\dot{\boldsymbol{x}} \mid v(\dot{\boldsymbol{x}})=<v_{\text {max }}(\dot{\boldsymbol{x}})\right\} \bigcup\left\{\dot{\boldsymbol{x}} \mid v(\dot{\boldsymbol{x}})>=v_{\text {min }}(\dot{\boldsymbol{x}})\right\}
$$


where $v(\dot{\boldsymbol{x}})$ is the biped walking speed, and $v_{\text {max }}(\dot{\boldsymbol{x}})$ and $v_{\text {min }}(\dot{\boldsymbol{x}})$ are the maximum and minimum values that have been proved to allow at least one walking cycle, respectively.

Definition 2 (Basin of Fall). Let $\boldsymbol{B} \in \mathbf{R}^{\mathrm{N}}$ be a subset of the biped walking state space. $\forall \boldsymbol{x} \in \boldsymbol{B} \Rightarrow$ $\exists \Delta t \geq 0$ s.t. $\boldsymbol{x}(t+\Delta t) \in \boldsymbol{Q}_{F a l l}$, where $\boldsymbol{B}$ is called the Basin of Fall.

Definition 3 (Stable). Biped walking is stable if the state space of walking motion does not intersect with $\boldsymbol{B}$.

\subsection{Control Strategy on Compliant Slopes}

As per the definition of stability, stable walking on compliant slopes is achieved by adjusting the speed of walking within a certain range. On the basis of observation of human walking characteristics, that is, the speed of the body increases when it leans forward and decreases automatically when it leans backward, walking speed is regulated by controlling centroid displacement. Therefore, a feedforward control strategy based on the motion state of the CoM is proposed to stabilize the entire walking process on compliant slopes. The horizontal velocity of the robot's CoM $u_{\mathrm{f}}$ at the end of the SSP is considered to be the system output, and the final displacement of the CoM $x_{\mathrm{f}}$ at the end of the SSP is selected to be the system input. Thus, a single-input-single-output (SISO) control system is developed in which $x_{\mathrm{f}}$ is the controlled input and $u_{\mathrm{f}}$ is the controlled output. On the basis of the state in the current cycle, $x_{\mathrm{f}}$ is calculated to regulate the $u_{\mathrm{f}}$ of next cycle to track the ideal horizontal velocity of the CoM $u_{\mathrm{fd}}$, and stable walking is realized.

\subsubsection{Model Equivalence}

To identify the relationship between $u_{\mathrm{f}}$ and $x_{\mathrm{f}}$, an equivalent rod-ground coupling model based only on the robot's CoM was established, as shown in Fig. 2. Moreover, two submodels were constructed in sequence - the impact submodel and the swing submodel—corresponding to the two 
phases of a full walking cycle. Detection of the terminal time of the impact phase is a substantial challenge for the equivalent model. To consider the actual effect of the ground compliance on the robot's CoM motion during a DSP, a coefficient $\lambda$ is introduced as an indicator. This coefficient is used to measure the decrease in the tangential contacting velocity at the rod-ground CP. When the dissipation ratio of the tangential contact velocity is equal or less than $\lambda$, the rod enters into the swing submodel.

The calculation process comprises two steps. First, the final state of the rod's CoM only under a tangential force of the ground is derived. Subsequently, the state is modified using the mean value theorem. The final states of the rod's CoM only under the tangential force are derived as

$$
\left\{\begin{array}{c}
\hat{u}\left(t_{1}\right)=(1-\lambda) \hat{u}_{\mathrm{i}} \\
\tilde{\hat{v}}\left(t_{1}\right)=v_{\mathrm{i}}+\tilde{\omega}\left(t_{1}\right) x_{\mathrm{i}} \\
\tilde{\omega}\left(t_{1}\right)=\omega_{\mathrm{i}}+\lambda y_{\mathrm{i}} \hat{u}_{\mathrm{i}} /\left(\rho^{2}+y_{\mathrm{i}}^{2}\right)
\end{array}\right.
$$

where $u$ and $v$ denote the horizontal and vertical velocity of CoM; $\omega$ is the rotational angular velocity of the rod's CoM; $\hat{u}$ and $\hat{v}$ are the tangential and normal components of relative velocity at the rod-ground $\mathrm{CP}$, respectively; $x$ and $y$ are the horizontal and vertical displacements of the $\mathrm{CoM}$ to the $\mathrm{CP}$ at the initial time of impact, respectively; $\rho$ is the gyration radius of the rod; the subscript "i" denotes the initial value; the superscript " $\sim$ " denotes the state that is only under the horizontal ground reaction force; and $t_{1}$ denotes the terminal time of impact submodel. Then, according to the theorem of impulse, $t_{1}$ is expressed as

$$
t_{1}=\frac{m_{\mathrm{t}} \rho^{2} \lambda}{c\left(\rho^{2}+y_{\mathrm{i}}^{2}\right)}
$$

where $m_{\mathrm{t}}$ is the robot's total mass.

Second, a normal force of the ground is added to modify the states of the rod's CoM. To realize at least one walking cycle, $\omega(t)$ must be nonnegative and $\hat{v}_{\mathrm{i}} \leq \hat{v}(t) \leq \tilde{v}\left(t_{1}\right)$ must be set. The final states of the rod's CoM are modified as 


$$
\left\{\begin{array}{l}
u=u_{\mathrm{i}}-\lambda \frac{\rho^{2}}{\rho^{2}+y_{\mathrm{i}}^{2}} \hat{u}_{\mathrm{i}} \\
v=v_{\mathrm{i}}-\frac{\rho^{2} \lambda\left[2 \hat{v}_{\mathrm{i}}\left(\rho^{2}+y_{\mathrm{i}}^{2}\right)+\hat{u}_{\mathrm{i}} x_{\mathrm{i}} y_{\mathrm{i}} \lambda\right]}{2\left(\rho^{2}+y_{\mathrm{i}}^{2}\right)^{2}} \\
\omega=\omega_{\mathrm{i}}-\lambda \frac{v_{\mathrm{i}} x_{\mathrm{i}}-u_{\mathrm{i}} y_{\mathrm{i}}+\omega_{\mathrm{i}}\left(x_{\mathrm{i}}^{2}+y_{\mathrm{i}}^{2}\right)}{\rho^{2}+y_{\mathrm{i}}^{2}}+\frac{\hat{u}_{\mathrm{i}} x_{\mathrm{i}}^{2} y_{\mathrm{i}} \lambda^{2}}{2\left(\rho^{2}+y_{\mathrm{i}}^{2}\right)^{2}}
\end{array}\right.
$$

During a SSP, a passive inverted pendulum is used to formulate an equivalent swing model for the biped (Bazargan-Lari et al., 2015). A straight-line trajectory is designed for the inverted pendulum's CoM trajectory. The equivalent swing submodel is described in Fig. 2 (c). The total kinetic energy variation in this phase is given as

$$
\begin{gathered}
\Delta W=(1-\lambda) \int_{\theta_{\mathrm{i}}}^{\theta_{\mathrm{f}}} f d r=(1-\lambda) \int_{\theta_{\mathrm{i}}}^{\theta_{\mathrm{f}}} \frac{m_{\mathrm{t}} g}{\cos \theta} d\left(\frac{y_{\mathrm{i}}}{\cos \theta}\right) \\
=(1-\lambda) \frac{m_{\mathrm{t}} g\left(x_{\mathrm{f}}^{2}-x_{\mathrm{i}}^{2}\right)}{2 y_{\mathrm{i}}}
\end{gathered}
$$

where $\theta_{\mathrm{i}}$ and $\theta_{\mathrm{f}}$ denote the initial and final angles of a swing phase, respectively.

After the whole equivalent model, the kinetic energy of the rod's CoM at the end of a full cycle is calculated to be

$$
E=K e_{\mathrm{f}}+\Delta W
$$

where $K e_{\mathrm{f}}=m_{\mathrm{t}}\left(u^{2}+v^{2}+\rho^{2} \omega^{2}\right) / 2$. Therefore, $u_{\mathrm{f}}$ at the terminal time of the equivalent cycle is obtained using the following equation, which is detailed in the Appendix.

$$
\begin{aligned}
u_{\text {fcal }} & =U_{\text {rod }}\left(\lambda, v_{\mathrm{i}}, u_{\mathrm{i}}, x_{\mathrm{i}}, x_{\mathrm{f}}, y_{\mathrm{i}}\right) \\
& =\sqrt{3 E / 2 m_{\mathrm{t}}} y_{\mathrm{i}} / \sqrt{y_{\mathrm{i}}^{2}+x_{\mathrm{f}}^{2}}
\end{aligned}
$$

\subsubsection{Implementation of the control strategy}

A control scheme was developed to implement the control strategy, as shown in Fig. 3, where $\boldsymbol{q}_{\mathrm{r}}$, $\dot{\boldsymbol{q}}_{\mathrm{r}}, \quad \boldsymbol{q}_{\mathrm{c}}$, and $\dot{\boldsymbol{q}}_{\mathrm{c}}$ denote the practical position, practical velocity, ideal position, and ideal velocity of the robot's joints, respectively; $j$ denotes the number of cycles; and $i \in\{1,2, \ldots . ., N\}$ is the 
sampling point during a cycle. First, the speed of the CoM is obtained using the robot state in the $j-1$ th cycle. Second, the attenuation coefficient $\lambda$ is calculated according to $u_{\mathrm{f}}$. Then, the displacement of the CoM $x_{\mathrm{fc}}$ in the $j$ th cycle is calculated according to $\lambda$. Subsequently, the robot's gait is planned on the basis of $x_{\mathrm{fc}}$. Finally, the robot's walking is realized through tracking the ideal gait, and the final state of the robot in the current cycle is recorded for calculation in the next cycle.

For the prototype, $\boldsymbol{q}_{\mathrm{r}}$ is obtained from the angle sensor mounted on each joint, and $\dot{\boldsymbol{q}}_{\mathrm{r}}$ is obtained through a differential calculation. Based on the formula of the centroid calculation in theoretical mechanics, the position and velocity of the CoM are obtained as follows:

$$
\left\{\begin{array}{l}
x=\frac{\sum m_{\mathrm{k}} x_{\mathrm{k}}}{\Sigma m_{\mathrm{k}}}, y=\frac{\sum m_{\mathrm{k}} y_{\mathrm{k}}}{\Sigma m_{\mathrm{k}}} \\
u=\frac{\sum m_{\mathrm{k}} \dot{x}_{\mathrm{k}}}{\Sigma m_{\mathrm{k}}}, \quad v=\frac{\sum m_{\mathrm{k}} \dot{y}_{\mathrm{k}}}{\sum m_{\mathrm{k}}}
\end{array}\right.
$$

where $k \in\{1,2, \ldots ., 7\}$ denote the calf in the support phase, thigh in the support phase, thigh in the swing phase, calf in the swing phase, knee in the support phase, hip in both phases, and knee in the swing phase, respectively; $m_{\mathrm{k}}, x_{\mathrm{k}}, y_{\mathrm{k}}, \dot{x}_{\mathrm{k}}$, and $\dot{y}_{\mathrm{k}}$ denote the mass of the CoM, horizontal displacement of the CoM, vertical displacement of the CoM, horizontal velocity of the CoM, and vertical velocity of the CoM corresponding to $k$, respectively. The controller collects $x, y, u$, and $v$ at the initial state and final state of the SSP and denotes these parameters as $v_{\mathrm{i}}, u_{\mathrm{i}}, x_{\mathrm{i}}, x_{\mathrm{f}}$, $y_{\mathrm{i}}$, and $u_{\mathrm{f}}$. On the basis of equation (12), the linearization at point $\lambda=0$ can be solved by varying $\lambda$

$$
\begin{aligned}
\lambda & =\lambda\left(v_{\mathrm{i}}, u_{\mathrm{i}}, x_{\mathrm{i}}, x_{\mathrm{f}}, y_{\mathrm{i}}, u_{\mathrm{f}}\right) \\
& =\frac{u_{\mathrm{f}}-U_{\text {rod }}\left(0, v_{\mathrm{i}}, u_{\mathrm{i}}, x_{\mathrm{i}}, x_{\mathrm{f}}, y_{\mathrm{i}}\right)}{\left.\frac{\partial}{\partial \lambda} U_{\text {rod }}\left(\lambda, v_{\mathrm{i}}, u_{\mathrm{i}}, x_{\mathrm{i}}, x_{\mathrm{f}}, y_{\mathrm{i}}\right)\right|_{\lambda=0}}
\end{aligned}
$$

The displacement of the CoM $x_{\mathrm{fc}}$ for the next cycle is obtained by conducting first-order 
linearization at point $x=x_{\mathrm{i}}$,

$$
\begin{aligned}
x_{\mathrm{fc}}= & \chi\left(\lambda, \eta, v_{\mathrm{f}}, u_{\mathrm{f}}, x_{\mathrm{i}}, y_{\mathrm{f}}, L_{\text {stepd }}, u_{\mathrm{fd}}\right)=x_{\mathrm{f}}+\eta \Delta x_{\mathrm{f}} \\
= & x_{\mathrm{f}}+\eta \frac{u_{\mathrm{fd}}-U_{\text {rod }}\left(\lambda, v_{\mathrm{f}}, u_{\mathrm{f}}, x_{\mathrm{i}}, L_{\text {stepd }}-x_{\mathrm{i}}, y_{\mathrm{f}}\right)}{\left.\frac{\partial}{\partial x} U_{\text {rod }}\left(\lambda, v_{\mathrm{f}}, u_{\mathrm{f}}, x_{\mathrm{i}}, L_{\text {stepd }}-x_{\mathrm{i}}, y_{\mathrm{f}}\right)\right|_{x=x_{\mathrm{i}}}}
\end{aligned}
$$

where $L_{\text {stepd }}$ is the ideal step length for the control cycle, $x_{\mathrm{f}}$ is the horizontal displacement of the $\mathrm{CoM}$ in the current cycle, $\Delta x_{\mathrm{f}}$ is the ideal change in the displacement required to realize speed tracking, and $\eta$ is the displacement correction factor related to the slope angle $\gamma$.

Suppose that $\boldsymbol{q}_{\mathrm{f}}=\left[q_{1 \mathrm{f}}, q_{2 \mathrm{f}}, q_{3 \mathrm{f}}, q_{4 \mathrm{f}}\right]^{\prime}$ is the ideal gait at the terminal time of the SSP. Then, the gait obtained by the controller is defined as $\boldsymbol{q}_{\mathrm{fc}}=\left[q_{1 \mathrm{fc}}, q_{2 \mathrm{fc}}, q_{3 \mathrm{fc}}, q_{4 \mathrm{fc}}\right]^{\prime}$. Forward kinematics is used to calculate $x_{\mathrm{fc}}$ as follows:

$$
x_{\mathrm{fc}}=f_{\boldsymbol{q}_{\mathrm{f}} \rightarrow x_{\mathrm{f}}}\left(\boldsymbol{q}_{\mathrm{fc}}\right)
$$

Calculating $x_{\mathrm{fc}}$ to obtain $\boldsymbol{q}_{\mathrm{fc}}$ by using inverse kinematics is a multi-solution problem, and the following constraints are introduced to solve this problem.

$$
\left\{\begin{array}{l}
q_{2 \mathrm{fc}}=q_{2 \mathrm{f}}+\Delta q \\
q_{4 \mathrm{fc}}=q_{4 \mathrm{f}}+\Delta q
\end{array}\right.
$$

then

$$
\begin{gathered}
\Delta q=\frac{x_{\mathrm{fc}}-x_{\mathrm{f}}}{f_{\boldsymbol{q}_{\mathrm{f}} \rightarrow x_{\mathrm{f}}}\left(\boldsymbol{q}_{\mathrm{f}}+\Delta q\right) /\left.\Delta q\right|_{\Delta q=0}} \\
l_{\mathrm{st}}=\sqrt{2 l^{2}+2 l^{2} \cos \left(q_{2 \mathrm{fc}}\right)} l_{\mathrm{sw}}=\sqrt{2 l^{2}+2 l^{2} \cos \left(q_{4 \mathrm{fc}}\right)} \\
q_{3 \mathrm{fc}}=\arccos \frac{l_{\mathrm{st}}^{2}+l_{\mathrm{sw}}^{2}-L_{\mathrm{step}}^{2}}{2 l_{\mathrm{st}} l_{\mathrm{sw}}}
\end{gathered}
$$

After determining the terminal gait of the SSP, gait planning and motion control are required. The method of virtual constraints is used here, and holonomic constraints are imposed on the robot's configuration by using feedback control (Chevallereau et al., 2009). The walking pattern can be 
generated with holonomic constraints as follows:

$$
\boldsymbol{e}=\boldsymbol{h}(\boldsymbol{q})=\boldsymbol{q}_{\alpha}-\boldsymbol{h}_{\mathrm{d}}\left(q_{1}\right)
$$

where $\boldsymbol{e}$ is the $(3 \times 1)$ error between the actual value and the expected value of the virtual constraint; $\boldsymbol{q}_{\alpha}$ is the vector of control joints with virtual constraints, where $\alpha \in\{2,3,4\}$; and $\boldsymbol{h}_{\mathrm{d}}\left(q_{1}\right)$ is the $(3 \times 1)$ actuated variable vector varying as a function of $q_{1}$. Then, third-degree Bezier polynomials are used to generate an actuated joint trajectory.

On the basis of equation (6), the control torque that is required to remain on the virtual constraint surface corresponding to $\boldsymbol{q}_{\alpha}=\boldsymbol{h}_{\mathrm{d}}\left(q_{1}\right)$ can be computed as

$$
\boldsymbol{u}=\left(\frac{\partial \boldsymbol{h}(\boldsymbol{q})}{\partial \boldsymbol{q}} \boldsymbol{D}^{-1}(\boldsymbol{q}) \boldsymbol{B}_{2}\right)^{-1}\left(\frac{\partial^{2} \boldsymbol{h}_{d}\left(q_{1}\right)}{\partial q_{1}^{2}} \dot{q}_{1}^{2}(t)+\frac{\partial \boldsymbol{h}(\boldsymbol{q})}{\partial \boldsymbol{q}} \boldsymbol{D}^{-1}(\boldsymbol{q}) \boldsymbol{H}(\boldsymbol{q}, \dot{\boldsymbol{q}})\right)
$$

The feedback control variable is introduced to minimize the system modeling errors and external disturbances. The state of the robot on the constraint surface is achieved through input-output linearization. The torque of the actuated joints $\boldsymbol{u}^{*}$ is calculated as

$$
\boldsymbol{u}^{*}=\boldsymbol{u}-\left(\frac{\partial \boldsymbol{h}}{\partial \boldsymbol{q}} \boldsymbol{D}^{-1} \boldsymbol{B}_{2}\right)^{-1}\left(\frac{\boldsymbol{K}_{P}}{\varepsilon^{2}} \boldsymbol{e}+\frac{\boldsymbol{K}_{d}}{\varepsilon} \dot{\boldsymbol{e}}\right)(\varepsilon>0)
$$

which results in

$$
\ddot{\boldsymbol{e}}+\frac{\boldsymbol{K}_{d}}{\varepsilon} \dot{\boldsymbol{e}}+\frac{\boldsymbol{K}_{p}}{\varepsilon^{2}} \boldsymbol{e}=0
$$

where $\dot{\boldsymbol{e}}$ and $\ddot{\boldsymbol{e}}$ are the first- and second-order derivatives of $\boldsymbol{e}$, respectively; $\boldsymbol{K}_{p}$ and $\boldsymbol{K}_{d}$ are control parameter vectors; and $\varepsilon$ is a constant greater than zero.

\subsection{Control Strategy on Varying Compliant Slopes}

Realizing underactuated bipedal walking on varying slopes is crucial for the varying slopes in the natural environment. On the basis of further observation of human walking on varying slopes, a control strategy that achieves stabilization by adjusting the angle of the body (leaning forward or backward) to adapt to the varying slopes is proposed in this section. The controller differs in two 
respects from that employed to walk on a fixed slope. First, $\eta$ in equation (15) is determined for different slopes, and $\eta$ is adjusted according to the slope angle simultaneously. Then, the switching set $S$ in equation (5) must be regulated on the basis of the change in slope.

A schematic of the feedforward control on varying compliant slopes is shown in Fig. 4. For the prototype, the angle of the slope can be detected in advance. Suppose that the slope angle at the initial time of walking is detected as $\gamma_{0}$ by the sensor, and the slope angle is changed to $\gamma_{i}=\gamma_{0}+\delta_{i}$ at the segment $i$. In this paper, $\gamma_{i}$ is in the range of $\left[-10^{\circ}, 10^{\circ}\right]$. When a robot walks on varying compliant slopes, $\gamma$ in the kinetic equation (6) must be changed to $\gamma_{i}$, and the impact condition should also be modified. Consider the impact at $C_{2}$ as an example. If the angle of the slope does not change, then the swing leg comes in contact with line $P_{1} P_{2} x_{0}$. However, if the slope angle $\delta_{2}$ changes, then the swing leg comes in contact with line $P_{2} C_{2} x_{1}$ at $C_{2}$. The switching set $S$ is turned to detect the distance between the swing leg end and line $P_{2} C_{2} x_{1}$. As the change in slope is detected in advance, the equation of line $P_{2} C_{2} x_{1}$ can be obtained easily. The other parts of the controller are the same as those in the previous section.

During practical implementation, $u_{\mathrm{fd}}$ equals $u_{\mathrm{f}}$ in the first cycle. Thus, the first walking cycle is realized, and the walking process is stabilized by controlling the variation in the walking speed.

\section{Simulations and Discussion}

\subsection{The Determination of $\eta$}

The parameters of the robot's structure are shown in Table 1. The ground compliance parameters $k$ and $c$ are listed in Table 2. The optimal initial gait $(O I G)$ of the robot under a rigid robotground contact assumption is given as follows:

$$
\begin{aligned}
& \boldsymbol{q}_{\mathrm{f} 0}=[0.1814,0.2048,0.3050,-0.3358]^{\prime} \\
& \dot{\boldsymbol{q}}_{\mathrm{f} 0}=[2.5567,-0.1091,-1.8804,4.0332]^{\prime}
\end{aligned}
$$


The parameter $\eta$ in equation (14) must be determined in advance. However, it is very difficult to calculate the value of $\eta$ through analytical derivation. Therefore, a simulation experiment method and curve fitting were used to determine the value of $\eta$ in this study. First, the set of values of $\eta$ that can achieve stable walking on different slopes was determined through simulations. Then, the number of adjustment cycles and the speed change rate were used to optimize $\eta$. After the optimization process, the parameter set was reduced to a series of discrete points. Finally, $\eta$ was associated with the slope angle through curve fitting.

Let $G C 3$ be the parameter of ground compliance. The range $\left[-10^{\circ}, 10^{\circ}\right]$ is divided into 20 equal parts at intervals of $0.5^{\circ}$. Through simulation at the slope angles of $-10^{\circ},-9.5^{\circ},-9^{\circ} \ldots . .9 .5^{\circ}$, and $10^{\circ}, \eta$ for stable walking on the slopes is determined. After determining the range of $\eta$ for stable walking on different slopes, the optimal value of $\eta$ is selected. Statistics are calculated for 50 walking cycles. Define $N_{\Delta=2}$ as the number of adjustment cycles that have a speed error band of $2 \%$ and $M_{\mathrm{P}}$ as the speed overshoot; then, the objective function $\Gamma_{\mathrm{i}}$ is defined as follows. The smaller the value of $\Gamma_{\mathrm{i}}$, the more optimal the value of $\eta$ is.

$$
\Gamma_{\mathrm{i}}=\frac{N_{\Delta=2}}{51} \times 0.8+M_{\mathrm{P}} \times 0.2
$$

Taking $-6^{\circ}$ as an example, the robot's speed for different $\eta$ values is shown in Fig. 5. The objective function $\Gamma_{\mathrm{i}}$ was calculated using equation (23) to obtain the optimal value of $\eta$. The range of $\eta$ on the $-6^{\circ}$ slope is $(0,0.4]$, and the optimum value is 0.05 .

By using the same method, the ranges and optimal values of $\eta$ in $\left(-10^{\circ}, 10^{\circ}\right)$ were obtained, as shown in Fig. 6. The blue area in the figure is the desirable range of the value of $\eta$, the black solid line is the boundary of $\eta$, and red asterisk points are the optimal values of $\eta$. The optimal trajectory line of $\eta$ was obtained through interpolation between the red asterisk points.

\subsection{Walking on Fixed Slopes with Various Compliance Parameters}

The simulation experiment was conducted on fixed slopes with various compliance parameters. 
The compliance parameters are listed in Table 2. The processes of change in $u_{\mathrm{f}}$ for $10^{\circ}$ and $-10^{\circ}$ slopes without a controller are shown in Fig. 7 and Fig. 8, respectively. The results show the following:

1) The robot is unstable without a controller under the $O I G$, and the influence of the ground compliance on the robot is not obvious.

2) When the robot is walking downhill without a controller, the gravity potential is converted into kinetic energy. Moreover, $u_{\mathrm{f}}$ increases continually, and the robot is unstable after four or five walking cycles. When the robot is walking uphill, $u_{\mathrm{f}}$ decreases continually, thus making the robot unstable.

Then, the simulation experiments were implemented using the controller. Steady-state errors in $u_{\mathrm{f}}$ with different compliance parameters are listed in Tables 3 and 4. The processes of change in $u_{\mathrm{f}}$ are shown in Figs. 9 and 10. The results demonstrate the following:

1) When the controller is used, $u_{\mathrm{f}}$ can converge to a single value in the first cycle at various compliance values, which is consistent with the definition of underactuated bipedal stable walking.

2) When the robot is walking uphill, it requires more adjustment cycles than when it is walking downhill to obtain a single value of $u_{\mathrm{f}}$. The robot is stable after 20 cycles at $-10^{\circ}$, but it requires at least 30 cycles to achieve stability at $10^{\circ}$.

3) The effect of the damping $c$ is greater than that of the stiffness $k$ during walking. For a fixed parameter $c$, the stable error in $u_{\mathrm{f}}$ does not change substantially as $k$ is varied. By contrast, for a fixed value of $k$, the stable error in $u_{\mathrm{f}}$ changes more when $c$ is varied, especially when the robot is walking uphill.

4) The smaller the value of $c$, the greater is the fluctuation in $u_{\mathrm{f}}$ and the number of cycles required to attain stability.

The robot's joint angles and joint velocities versus time on the $-10^{\circ}$ slope at the ground 
compliance $G C 3$ are presented in Figs. 11 and 12, respectively. Fig. 13 displays the joint angles versus time on the $10^{\circ}$ slope at the ground compliance $G C 3$. The results show the following:

1) The gait is periodic after the robot is stabilized. Moreover, $q_{1}$ and $q_{3}$ increase monotonically in each walking cycle. During the SSP, the supporting leg remains in a straight state, and $q_{2}$ is equal to 0 .

2) It can be seen from the Fig. 12 that due to the compliance of the ground, the DSP is not instantaneous, and a change in $\dot{q}_{2}$ and $\dot{q}_{4}$ is evident during this period.

\subsection{Walking on Varying Slopes with Fixed Compliance Parameters}

Simulations were conducted to demonstrate the performance of the controller on varying slopes. In a real robot walking system, the slope angle $\gamma$ can be detected by using sensors in advance. Here, three varying slope conditions were generated randomly in MATLAB by using RANDI, as illustrated in Table $5\left(\gamma_{0}-\gamma_{4}\right.$ correspond to downhill walking, $\gamma_{5}$ denotes flat ground, and $\gamma_{6}-\gamma_{9}$ correspond to uphill walking).

With $G C 3$ as the parameter of ground compliance, the simulation experiments were conducted under the three conditions. The results are displayed in Fig. 14 and demonstrate the following:

1) In a certain range, $u_{\mathrm{f}}$ always fluctuates, which is consistent with the definition of stability presented in this paper. The controller can adapt to the changes in the slopes, and thus, underactuated bipedal walking is achieved on varying slopes.

2) Although speed control is achieved by a controller, the fluctuation in $u_{\mathrm{f}}$ when the robot is walking uphill is greater than the fluctuation when it is walking downhill.

3) The controller input $x_{\mathrm{f}}$ and output $u_{\mathrm{f}}$ are positively correlated. When the controller detects that $u_{\mathrm{f}}$ is higher (lower) than $u_{\mathrm{fd}}, x_{\mathrm{f}}$ is reduced (increased) by the controller to regulate $u_{\mathrm{f}}$ to track $u_{\mathrm{fd}}$, and this is consistent with the control strategy.

Fig. 15 shows the robot's joint angles versus time with the slope condition $C D 2$ in Table 5. The 
following results were observed.

1) The posture of the robot constantly adjusts to the changes in the slope angle.

2) The tilt angle of the robot is greater when it is walking uphill, and $q_{1}$ during uphill walking is higher than that during downhill walking.

3) The variation in the range of $q_{2}$ and $q_{4}$ during uphill walking is greater than that during downhill walking, and the knee joint of the robot is more bent when it is walking uphill.

\subsection{Walking on Varying Slopes with Varying Compliance Parameters}

To further evaluate the effectiveness of the controller on varying slopes with varying compliance parameters, three simulation conditions were generated. The variation in the slope is shown in Fig. 16 (a). The changes in the slope compliance are shown in Fig. 16 (b). The transition of the controller output $u_{\mathrm{f}}$ during walking is shown in Fig. $16(\mathrm{c})$. The transition of the controller input $x_{\mathrm{f}}$ is shown in Fig. 16 (d). Underactuated bipedal walking is achieved on varying slopes at various compliance values, and $u_{\mathrm{f}}$ is always controlled within a certain range.

Fig. 17 shows the robot's joint angles versus time with the condition $C D 2$. The posture of the robot constantly adjusts to the changes in the slope angle and the ground compliance. The results are similar to those observed in the fixed compliance parameter environment shown in Fig. 15.

\section{Conclusion}

In this paper, a feedforward control strategy to stabilize underactuated bipedal walking on varying compliant slopes with a known angle of inclination is proposed.

1) A new definition of the stability of underactuated walking on the basis of the walking speed is proposed. Stable walking is achieved by adjusting the state of the robot outside the Basin of Fall.

2) On the basis of the stability definition and human gait characteristics, a feedforward control strategy based on the motion state of the CoM is proposed. A rod-slope model is used to simplify the robot-slope model. Underactuated bipedal walking is simplified by using a SISO system that utilizes 
a polynomial with a definite number of degrees, and the control input is obtained by inverting the polynomial. The control algorithm could be applied easily to the prototype, and good real-time performance could be obtained.

3) The control strategy proposed in this paper can effectively stabilize underactuated bipedal walking on varying compliant slopes with a known angle of inclination through simulations.

Future work will be focused on two points: 1) realization of the control strategy on the prototype robot shown in Fig. 18 through obtaining the slope angle by using ultrasonic sensors and 2) development of effective methods to stabilize underactuated bipedal walking on varying compliant slopes without using the state variables of the slope angle.

\section{Acknowledgement}

This research is supported by National Natural Science Foundation of China (NSFC, Grant No. 51675385 and No. 51175383).

\section{References}

Bazargan-Lari, Y., Eghtesad, M., Khoogar, A.R., and Mohammad-Zadeh, A. 2015. Adaptive neural network control of a human swing leg as a double-pendulum considering self-impact joint constraint. Trans. Can. Soc. Mech. Eng. 39(2): 201-219. Available from http://www.tcsme.org/Papers/Vol39/Vol39No2Paper5.pdf.

Chemori, A., Floch, S.L., Krut, S., and Dombre, E. 2010. A control architecture with stabilizer for 3D stable dynamic walking of SHERPA biped robot on compliant ground. In Proceedings of the 10th IEEE-RAS International Conference on Humanoid Robots, Nashville, TN, USA, 6-8 December 2010. Univ. Montpellier 2 - CNRS, Montpellier, France. pp. 480-485.

Chen, C.Y., and Huang, P.H. 2012. Review of an autonomous humanoid robot and its mechanical control. J. VIB. CONTROL. 18(7): 973-982. dio: 10.1177/1077546310395974.

Chevallereau, C., Grizzle, J.W., and Shih, C.L. 2009. Asymptotically stable walking of a five-link underactuated 3-D bipedal robot. IEEE. T. ROBOT. 25(1): 37-50. dio: 10.1109/tro.2008.2010366.

Chu, L.M., Lin, J.R., Hsu, H.C., and Chang, Y.P. 2016. Effects of surface forces on squeeze EHL motion between elastic ball and elastic coated surface. Trans. Can. Soc. Mech. Eng. 40(5): 821-833. Available from 
http://www.tcsme.org/Papers/

Vo140/Vol40No5Paper13.pdf.

Collins, S., Ruina, A., Tedrake, R., and Wisse, M. 2005. Efficient bipedal robots based on passive-dynamic walkers. SCIENCE. 307(5712): 1082-1085. dio: 10.1126/science.1107799.

Grizzle, J.W., Abba, G., and Plestan, F. 2001. Asymptotically stable walking for biped robots: analysis via systems with impulse effects. IEEE. T. AUTOMAT. CONTR. 46(1): 51-64. dio: 10.1109/9.898695.

Grizzle, J.W., Chevallereau, C., Sinnet, R.W., and Ames, A.D. 2014. Models, feedback control, and open problems of 3D bipedal robotic walking. AUTOMATICA. 50(8): 1955-1988. dio: 10.1016/j.automatica.2014.04.021.

Hitomi, K., Shibata, T., Nakamura, Y., and Ishii, S. 2006. Reinforcement learning for quasi-passive dynamic walking of an unstable biped robot. ROBOT. AUTON. SYST. 54(12): 982-988. dio: 10.1016/j.robot.2006.05.014.

Hobbelen, D.G.E., and Wisse, M. 2007. A disturbance rejection measure for limit cycle walkers: the gait sensitivity norm. IEEE. T. ROBOT. 23(6): 1213-1224. dio: 10.1109/tro.2007.904908.

Park, H.W., Ramezani, A., and Grizzle, J.W. 2013. A finite-state machine for accommodating unexpected large ground-height variations in bipedal robot walking. IEEE. T. ROBOT. 29(2): 331-345. dio: 10.1109/tro.2012.2230992.

Plestan, F., Grizzle, J.W., Westervelt, E.R., and Abba, G. 2003. Stable walking of a 7-DOF biped robot. IEEE. T. ROBOT. AUTOM. 19(4): 653-668. dio: 10.1109/tra.2003.814514.

Shih, C.L., Grizzle, J.W., and Chevallereau, C. 2012. From stable walking to steering of a 3D bipedal robot with passive point feet. ROBOTICA. 30(7): 1119-1130. dio: 10.1017/s026357471100138x.

Spong, M.W., and Bullo, F. 2005. Controlled symmetries and passive walking. IEEE. T. AUTOMAT. CONTR. 50(7): 1025-1031.dio: 10.1109/tac.2005.851449.

Spong, M.W., Holm, J.K., and Lee, D. 2007. Passivity-based control of bipedal locomotion. IEEE. ROBOT. AUTOM. MAG. 14(2): 30-40. dio: 10.1109/mra.2007.380638.

Sreenath, K., Park, H.W., Poulakakis, I., and Grizzle, J.W. 2011. A compliant hybrid zero dynamics controller for stable, efficient and fast bipedal walking on MABEL. INT. J. ROBOT. RES. 30(9): 1170-1193. dio: $\underline{10.1177 / 0278364910379882 .}$.

Tao, G. 2014. Online regulation of the walking speed of a planar limit cycle walker via model predictive control. IEEE. T. IND. ELECTRON. 61(5): 2326-2333. dio: 10.1109/tie.2013.2272274.

Tedrake, R., Zhang, T.W., and Seung, H.S. 2004. Stochastic policy gradient reinforcement learning on a simple 3D biped. In Proceedings of the 18th IEEE-RSJ International Conference on Intelligent Robots and Systems, Sendai, Japan, 28 September - 2 October, 2004. Massachusetts Institute of Technology, Cambridge, MA. vol. 2843. pp. 2849-2854. 
Wang, T., Chevallereau, C., and Tlalolini, D. 2014. Stable walking control of a 3D biped robot with foot rotation. ROBOTICA. 32(4): 551-570. dio: 10.1017/s0263574713000866.

Wang, Y., Ding, J., and Xiao, X. 2017. An adaptive feedforward control method for under-actuated bipedal walking on the compliant ground. INT. J. ROBOT. AUTOM. 32(1): 63-77. dio: 10.2316/journal.206.2017.1.206-4779.

Westervelt, E.R., Grizzle, J.W., Chevallereau, C., Choi, J.H., and Morris, B. 2007. Feedback control of dynamic bipedal robot locomotion. Boca Raton, USA. CRC Press.

Westervelt, E.R., Grizzle, J.W., and Koditschek, D.E. 2003. Hybrid zero dynamics of planar biped walkers. IEEE. T. AUTOMAT. CONTR. 48(1): 42-56. dio: 10.1109/tac.2002.806653.

Yadukumar, S.N., Pasupuleti, M., and Ames, A.D. 2012. Human-inspired underactuated bipedal robotic walking with AMBER on flat-ground, up-slope and uneven terrain. In Proceedings of the 26th IEEE/RSJ International Conference on Intelligent Robots and Systems, Vilamoura, Algarve, Portugal, 7-12 October, 2012. Texas A\& M University, College Station, TX. pp. 2478-2483.

Yang, T., Westervelt, E.R., Serrani, A., and Schmiedeler, J.P. 2009. A framework for the control of stable aperiodic walking in underactuated planar bipeds. AUTON. ROBOT. 27(3): 277-290. dio: 10.1007/s10514-009-9126-y. 


\section{Tables}

Table 1. Structure parameters of the robot

\begin{tabular}{ccccc}
\hline parameters & $l(\mathrm{~m})$ & $m(\mathrm{~kg})$ & $m_{\mathrm{k}}(\mathrm{kg})$ & $m_{\mathrm{h}}(\mathrm{kg})$ \\
\hline values & 0.3 & 0.4 & 0.8 & 2.5 \\
\hline
\end{tabular}


Table 2. Properties of compliant ground

\begin{tabular}{ccc}
\hline & $k\left(\times 10^{4} \mathrm{~N} / \mathrm{m}\right)$ & $c\left(\times 10^{5} \mathrm{~N} \cdot \mathrm{s} / \mathrm{m}\right)$ \\
\hline$G C 1\left(k_{1}, c_{1}\right)$ & 6 & 8 \\
$G C 2\left(k_{2}, c_{2}\right)$ & 12 & 16 \\
$G C 3\left(k_{3}, c_{3}\right)$ & 24 & 32 \\
$\operatorname{GC} 4\left(k_{4}, c_{4}\right)$ & 48 & 64 \\
$\operatorname{GC5}\left(k_{5}, c_{5}\right)$ & 96 & 128 \\
\hline
\end{tabular}


Table 3. Stable error of $u_{\mathrm{f}}$ with different compliant parameters on a $-10^{\circ}$ slope

\begin{tabular}{cccccc}
\hline & $k_{1}$ & $k_{2}$ & $k_{3}$ & $k_{4}$ & $k_{5}$ \\
\hline$c_{1}$ & $0.59 \%$ & $0.58 \%$ & $0.58 \%$ & $0.59 \%$ & $0.57 \%$ \\
$c_{2}$ & $0.57 \%$ & $0.56 \%$ & $0.55 \%$ & $0.58 \%$ & $0.57 \%$ \\
$c_{3}$ & $0.53 \%$ & $0.53 \%$ & $0.52 \%$ & $0.52 \%$ & $0.52 \%$ \\
$c_{4}$ & $0.45 \%$ & $0.44 \%$ & $0.43 \%$ & $0.43 \%$ & $0.44 \%$ \\
$c_{5}$ & $0.28 \%$ & $0.25 \%$ & $0.24 \%$ & $0.23 \%$ & $0.23 \%$ \\
\hline
\end{tabular}


Table 4. Stable error of $u_{\mathrm{f}}$ with different compliant parameters on a $10^{\circ}$ slope

\begin{tabular}{cccccc}
\hline & $k_{1}$ & $k_{2}$ & $k_{3}$ & $k_{4}$ & $k_{5}$ \\
\hline$c_{1}$ & $1.45 \%$ & $1.46 \%$ & $1.45 \%$ & $1.43 \%$ & $1.48 \%$ \\
$c_{2}$ & $1.23 \%$ & $1.22 \%$ & $1.23 \%$ & $1.22 \%$ & $1.19 \%$ \\
$c_{3}$ & $0.61 \%$ & $0.61 \%$ & $0.60 \%$ & $0.59 \%$ & $0.53 \%$ \\
$c_{4}$ & $0.73 \%$ & $0.73 \%$ & $0.72 \%$ & $0.72 \%$ & $0.65 \%$ \\
$c_{5}$ & $0.88 \%$ & $0.88 \%$ & $0.87 \%$ & $0.88 \%$ & $0.79 \%$ \\
\hline
\end{tabular}


Table 5. Arrays of Randomly Generated Slopes

\begin{tabular}{cccccccc}
\hline & $C D 1$ & $C D 2$ & $C D 3$ & & $C D 1$ & $C D 2$ & $C D 3$ \\
\hline$\gamma_{0}\left({ }^{\circ}\right)$ & -2.81 & -2.55 & -1.76 & $\gamma_{5}\left({ }^{\circ}\right)$ & 0 & 0 & 0 \\
$\gamma_{1}\left({ }^{\circ}\right)$ & -4.40 & -2.24 & -7.22 & $\gamma_{6}\left({ }^{\circ}\right)$ & 5.82 & 7.58 & 4.98 \\
$\gamma_{2}\left(^{\circ}\right)$ & -5.27 & -6.68 & -4.73 & $\gamma_{7}\left({ }^{\circ}\right)$ & 5.41 & 7.43 & 9.60 \\
$\gamma_{3}\left({ }^{\circ}\right)$ & -4.57 & -8.44 & -1.52 & $\gamma_{8}\left({ }^{\circ}\right)$ & 8.70 & 3.92 & 3.40 \\
$\gamma_{4}\left({ }^{\circ}\right)$ & -8.75 & -3.45 & -3.41 & $\gamma_{9}\left({ }^{\circ}\right)$ & 2.65 & 6.55 & 5.85 \\
\hline
\end{tabular}




\section{Figure Captions}

Fig. 1. Mathematical model of a bipedal robot on a compliant slope

Fig. 2. Equivalent motion system on a compliant slope (tra1: a possible trajectory of the robot's CoM; tra2: the designed trajectory of the inverted pendulum's CoM)

Fig. 3. Feedforward control strategy

Fig. 4. Feedforward control on varying compliant slopes

Fig. 5. Evaluation of $u_{\mathrm{f}}$ with various values of $\eta$ at a slope angle of $-6^{\circ}$

Fig. 6. Ranges and optimal values of $\eta$

Fig. 7. Changes in $u_{\mathrm{f}}$ on a $-10^{\circ}$ slope without a controller

Fig. 8. Changes in $u_{\mathrm{f}}$ on a $10^{\circ}$ slope without a controller

Fig. 9. Changes in $u_{\mathrm{f}}$ on $\mathrm{a}-10^{\circ}$ slope with a controller

Fig. 10. Changes in $u_{\mathrm{f}}$ on a $10^{\circ}$ slope with a controller

Fig. 11. Joint angles with respect to time on a $-10^{\circ}$ slope with a controller

Fig. 12. Joint velocities with respect to time on a $-10^{\circ}$ slope with a controller

Fig. 13. Joint angles with respect to time on a $10^{\circ}$ slope with a controller

Fig. 14. Simulation results on varying slopes with fixed compliance parameters. (a) Changes in the slope angle (b) Changes in $u_{\mathrm{f}}$ with a controller (c) Changes in $x_{\mathrm{f}}$ with a controller

Fig. 15. Joint angles with respect to time on varying slopes with fixed compliance parameters

Fig. 16. Simulation results on varying slopes with varying compliance parameters. (a) Changes in the slope angle (b) Changes in the ground compliant (c) Changes in $u_{\mathrm{f}}$ with a controller (d) Changes in $x_{\mathrm{f}}$ with a controller

Fig. 17. Joint angles with respect to time on varying slopes with varying compliance 
parameters

Fig. 18. Prototype robot "UBbot" 


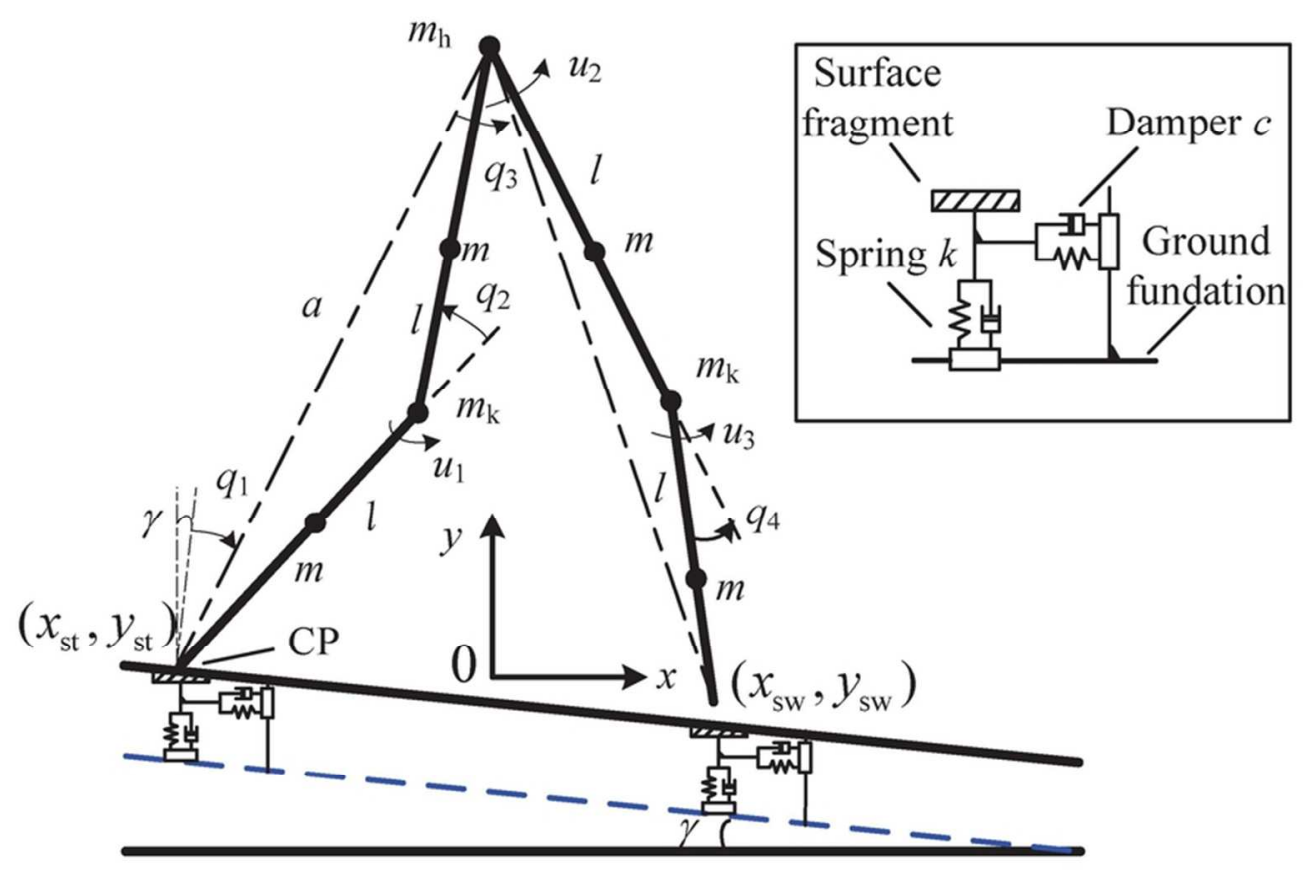

Fig. 1. Mathematical model of a bipedal robot on a compliant slope $75 \times 51 \mathrm{~mm}(300 \times 300 \mathrm{DPI})$ 


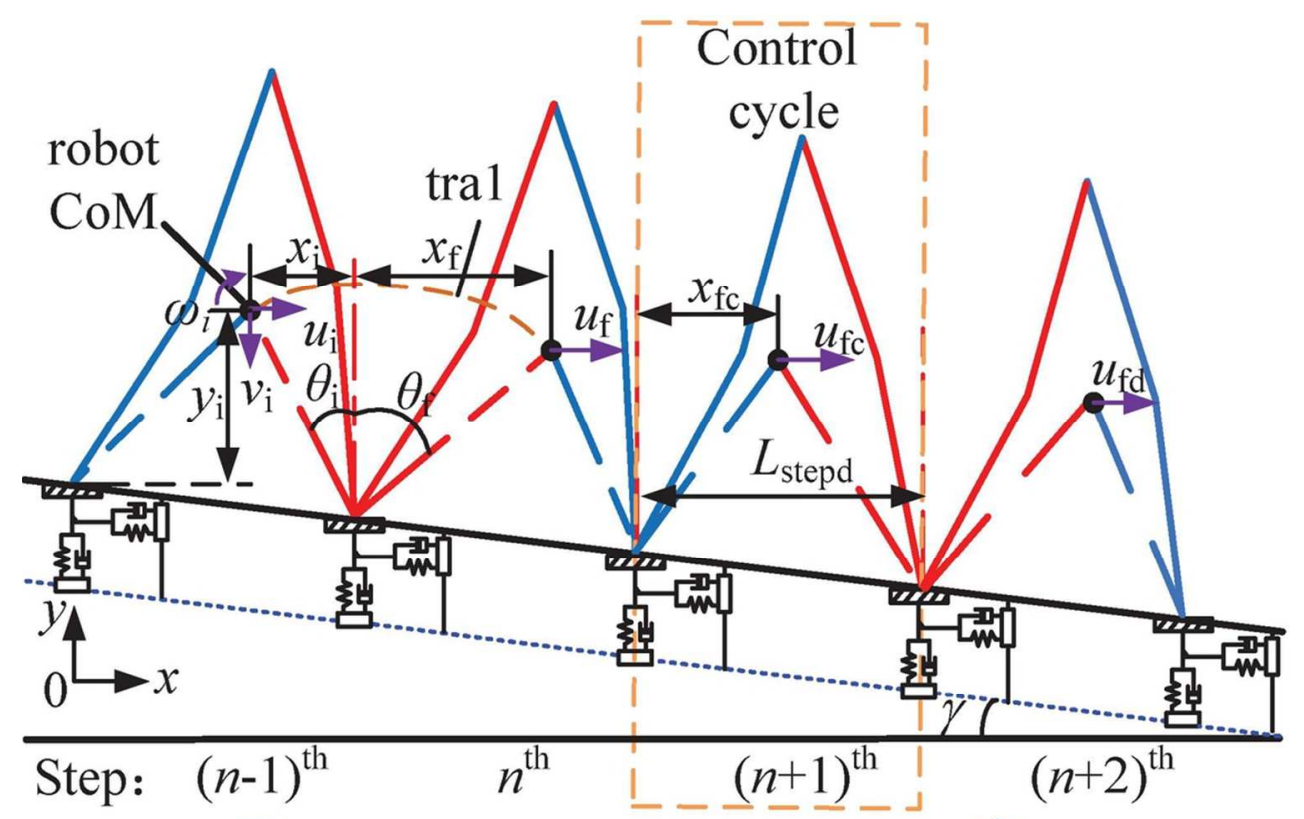

(a) Original walking system

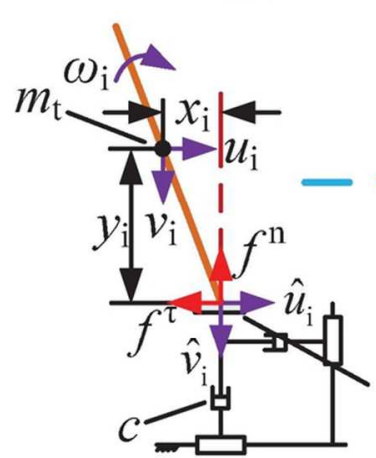

(b) Impact submodel

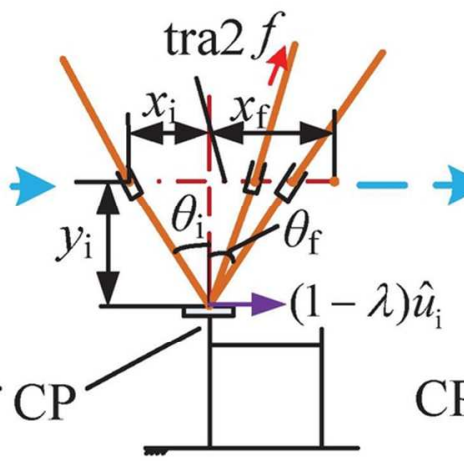

(c) Swing submodel

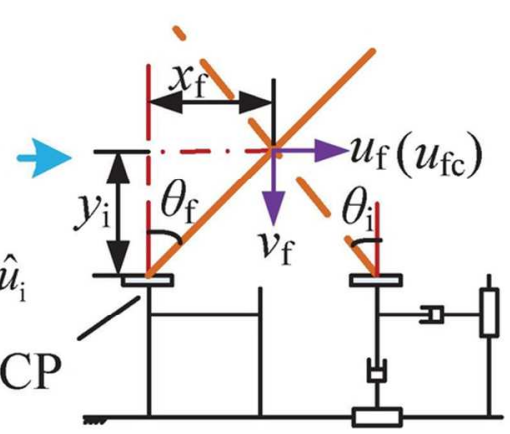

(d) Final state of overall model

Fig. 2. Equivalent motion system on a compliant slope $99 \times 114 \mathrm{~mm}(300 \times 300 \mathrm{DPI})$ 


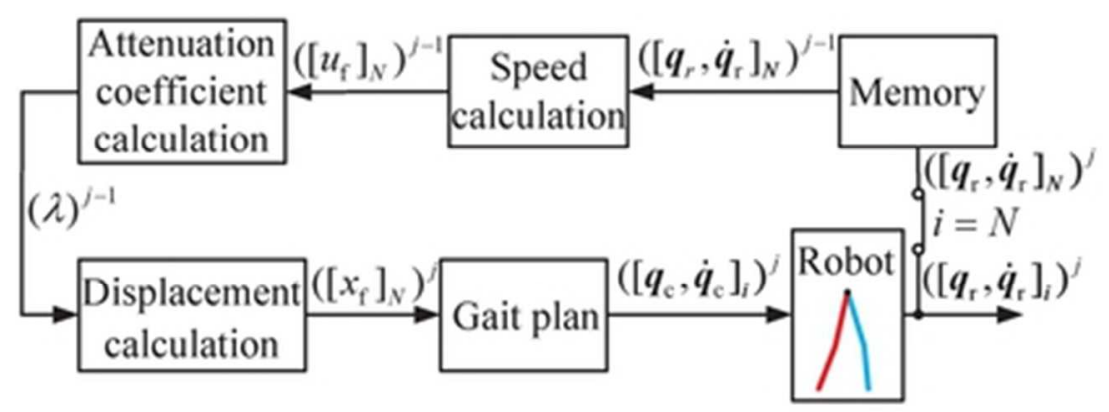

Fig. 3. Feedforward control strategy $35 \times 13 \mathrm{~mm}(300 \times 300$ DPI $)$ 


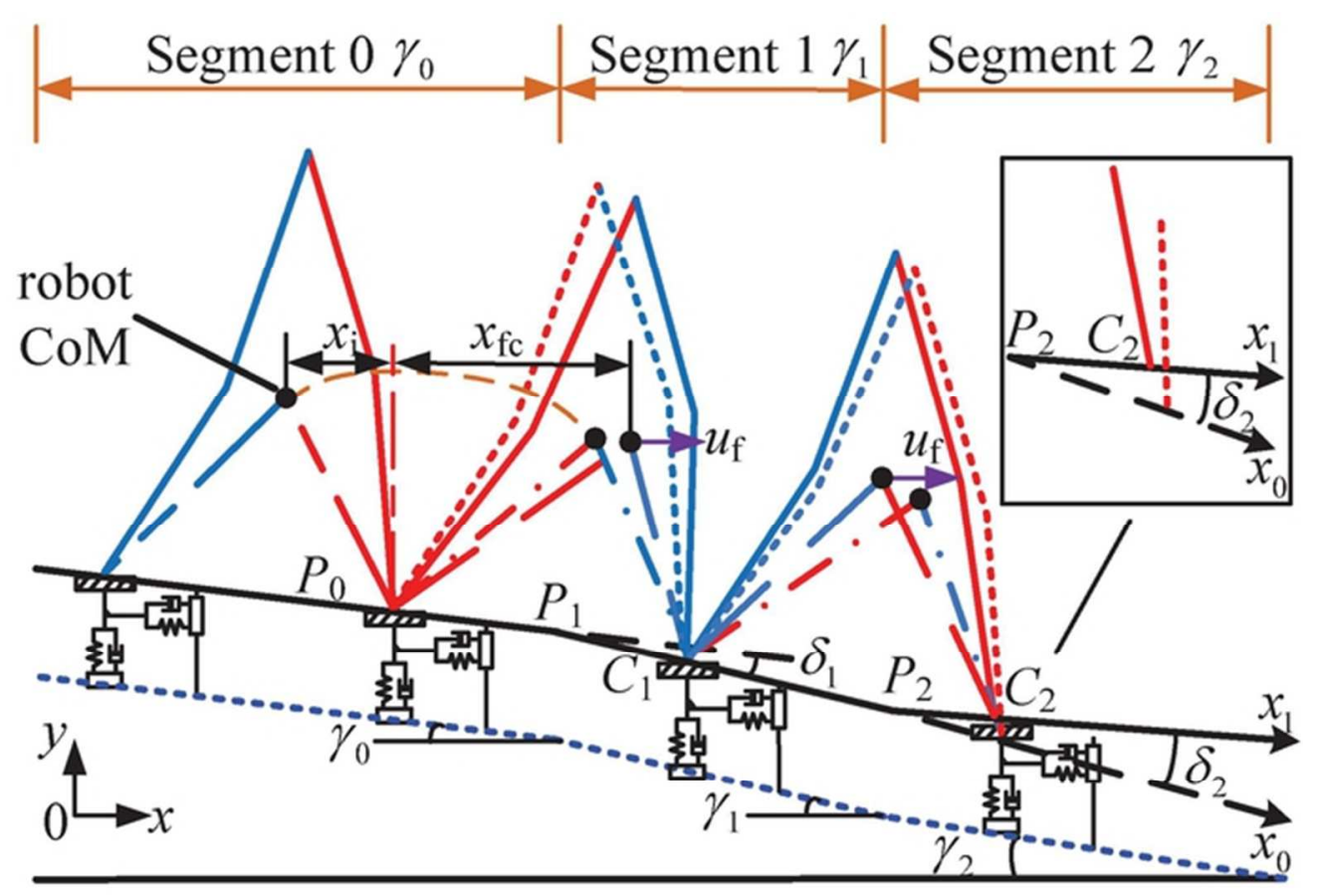

Fig. 4. Feedforward control on varying compliant slopes

$58 \times 40 \mathrm{~mm}(300 \times 300 \mathrm{DPI})$ 


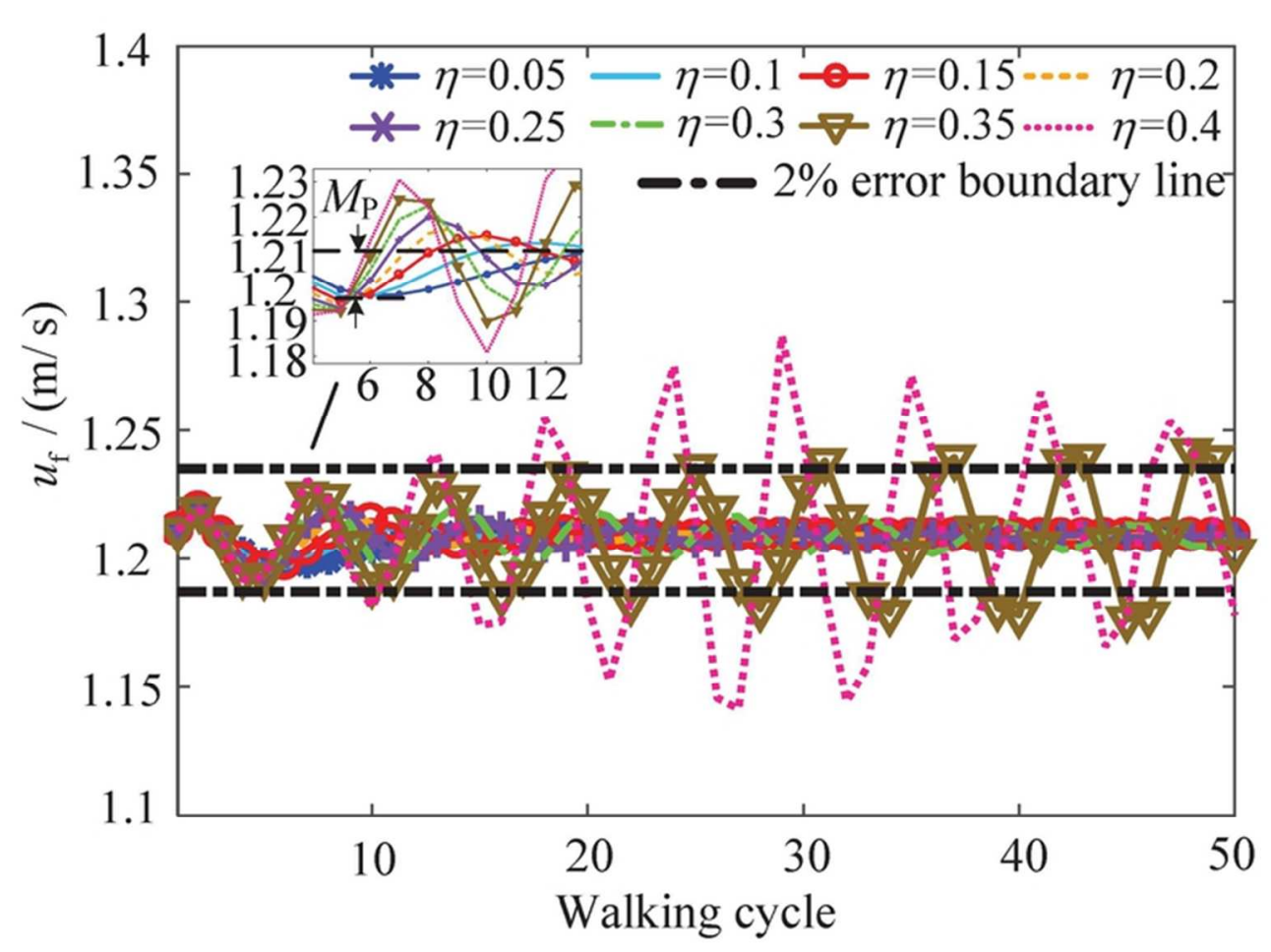

Fig. 5. Evaluation of uf with various values of $\eta$ at a slope angle of $-6^{\circ}$

$67 \times 49 \mathrm{~mm}(300 \times 300 \mathrm{DPI})$ 


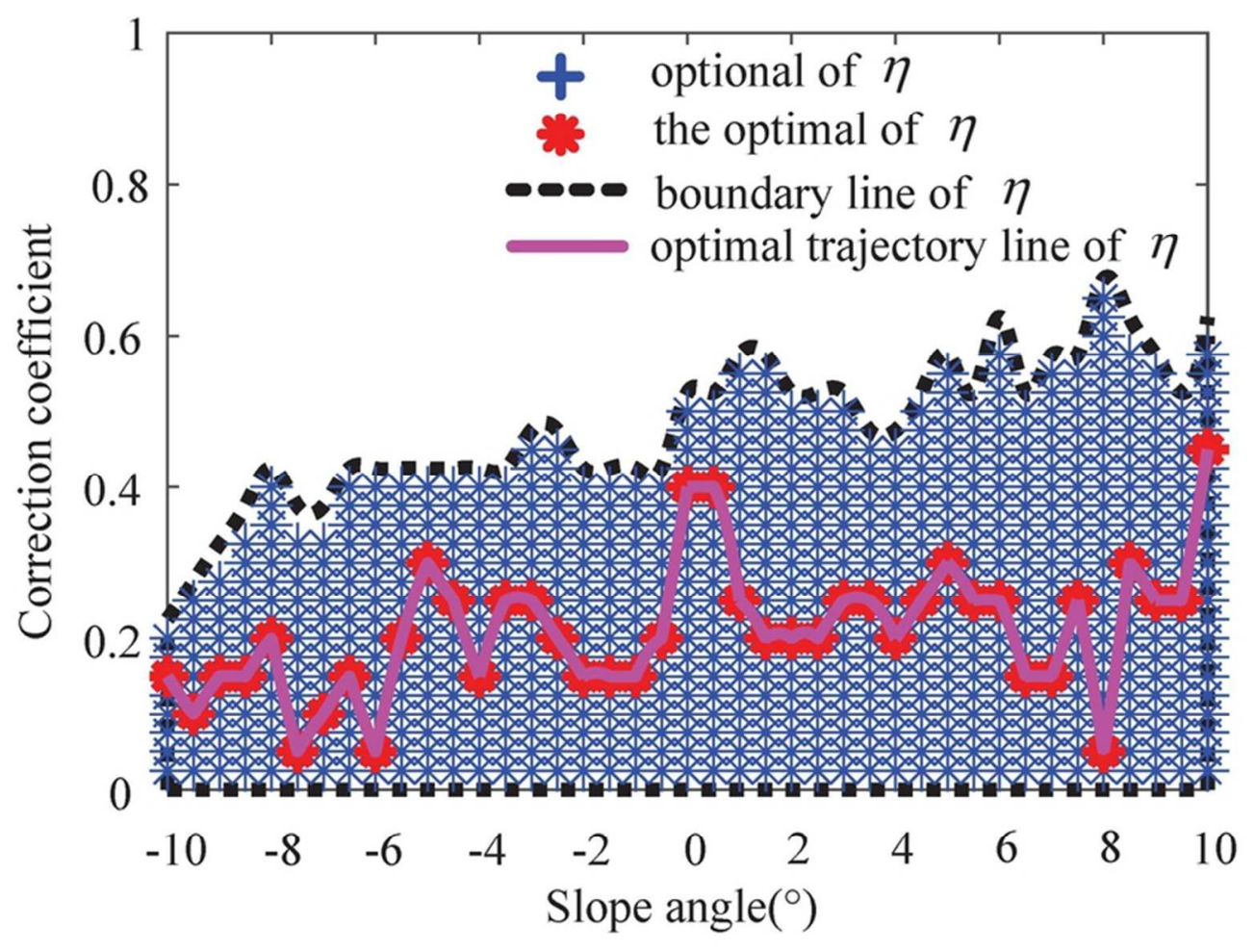

Ranges and optimal values of $\eta$

$71 \times 54 \mathrm{~mm}(300 \times 300$ DPI $)$ 

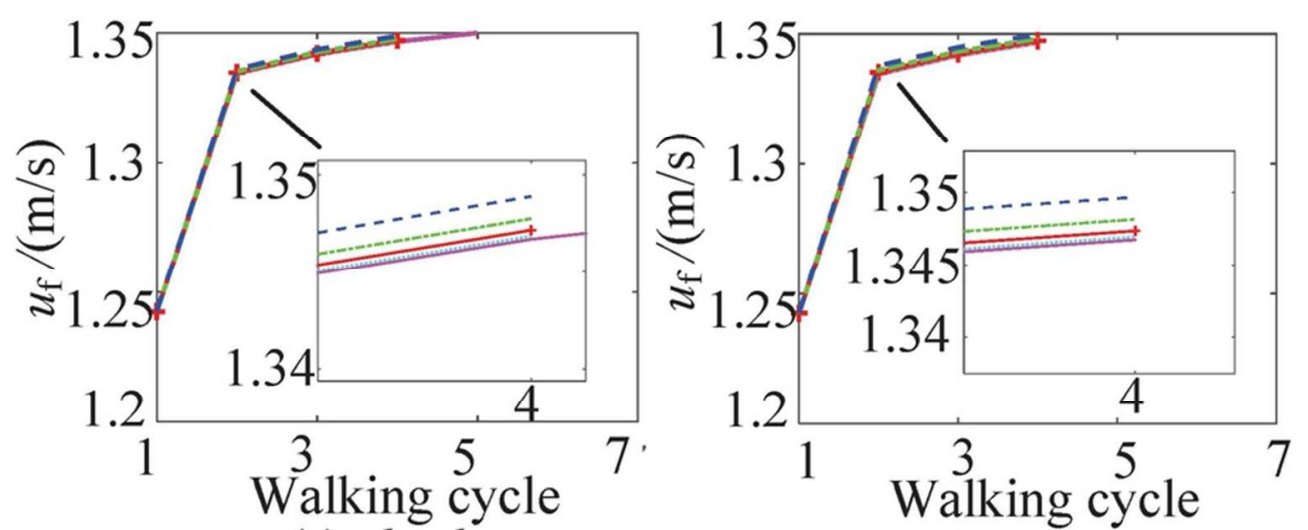

(a) $k=k_{1}$

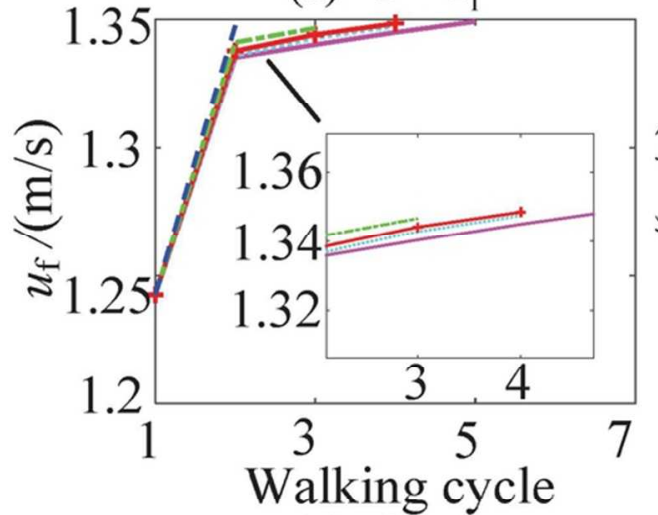

(c) $k=k_{4}$

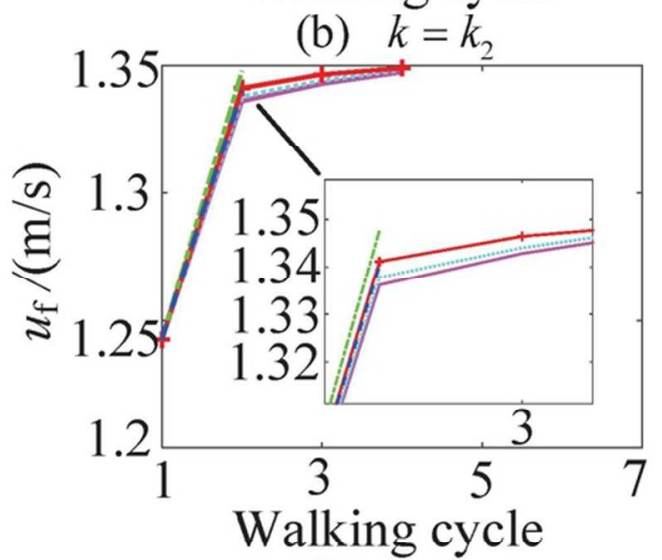

(b) $k=k_{2}$

(d) $k=k_{5}$

$\cdots c_{1}---c_{2}+c_{3}$

$c_{4} \quad-c_{5}$

Changes in uf on $\mathrm{a}-10^{\circ}$ slope without a controller

$77 \times 71 \mathrm{~mm}(300 \times 300$ DPI $)$ 

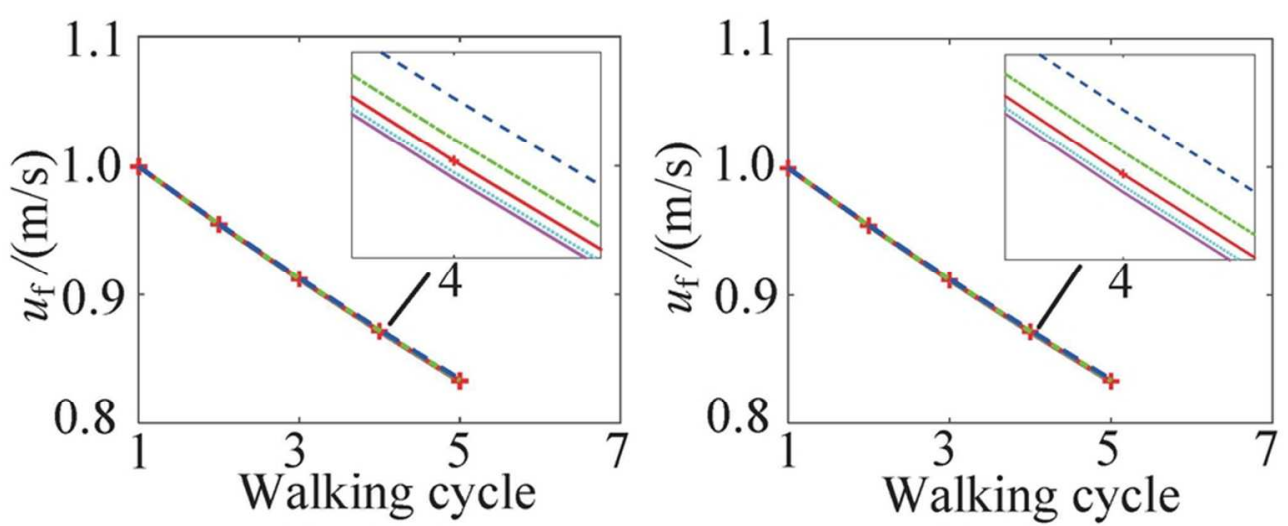

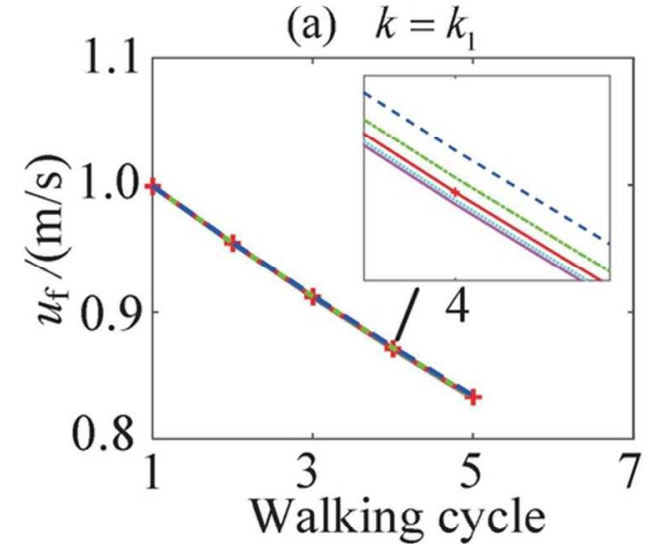

(c) $k=k_{4}$

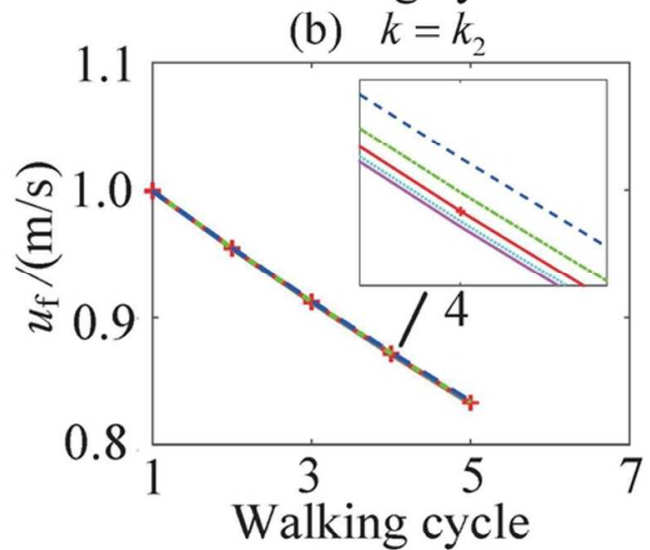

(d) $k=k_{5}$

$\cdots c_{1} \quad-\cdots c_{2}+c_{3}$

$\ldots c_{4}$

$c_{5}$

Changes in uf on a $10^{\circ}$ slope without a controller

$79 \times 71 \mathrm{~mm}(300 \times 300 \mathrm{DPI})$ 

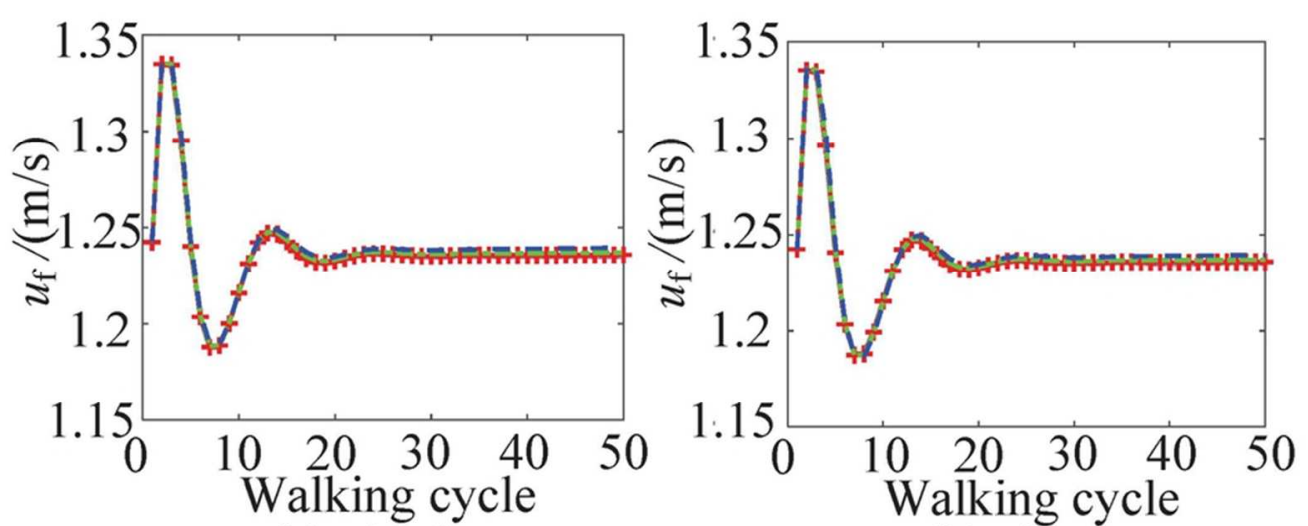

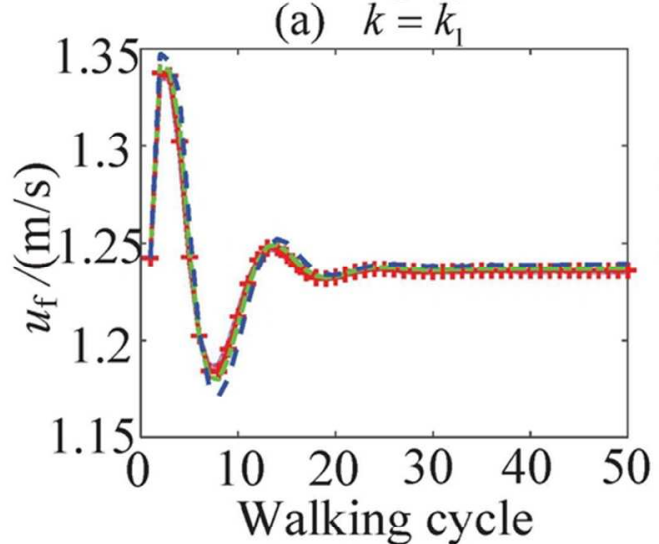

(c) $k=k_{4}$

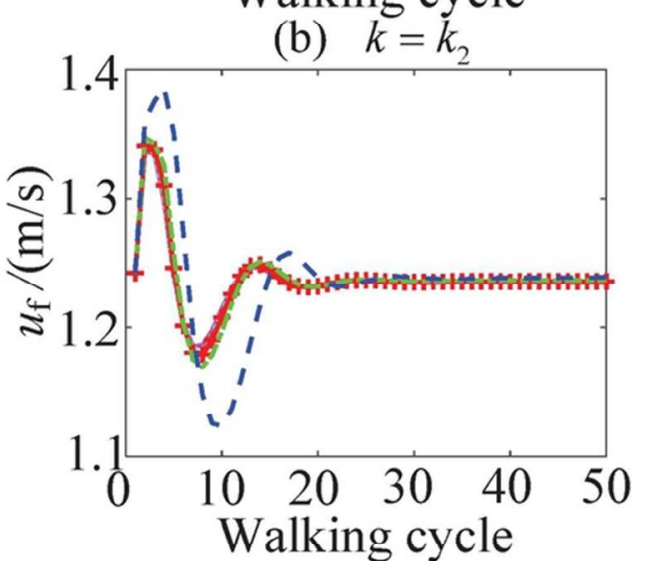

(d) $k=k_{5}$

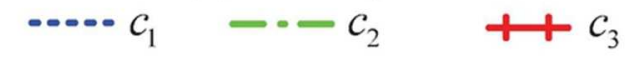

Changes in uf on $a-10^{\circ}$ slope with a controller

$79 \times 71 \mathrm{~mm}(300 \times 300 \mathrm{DPI})$ 

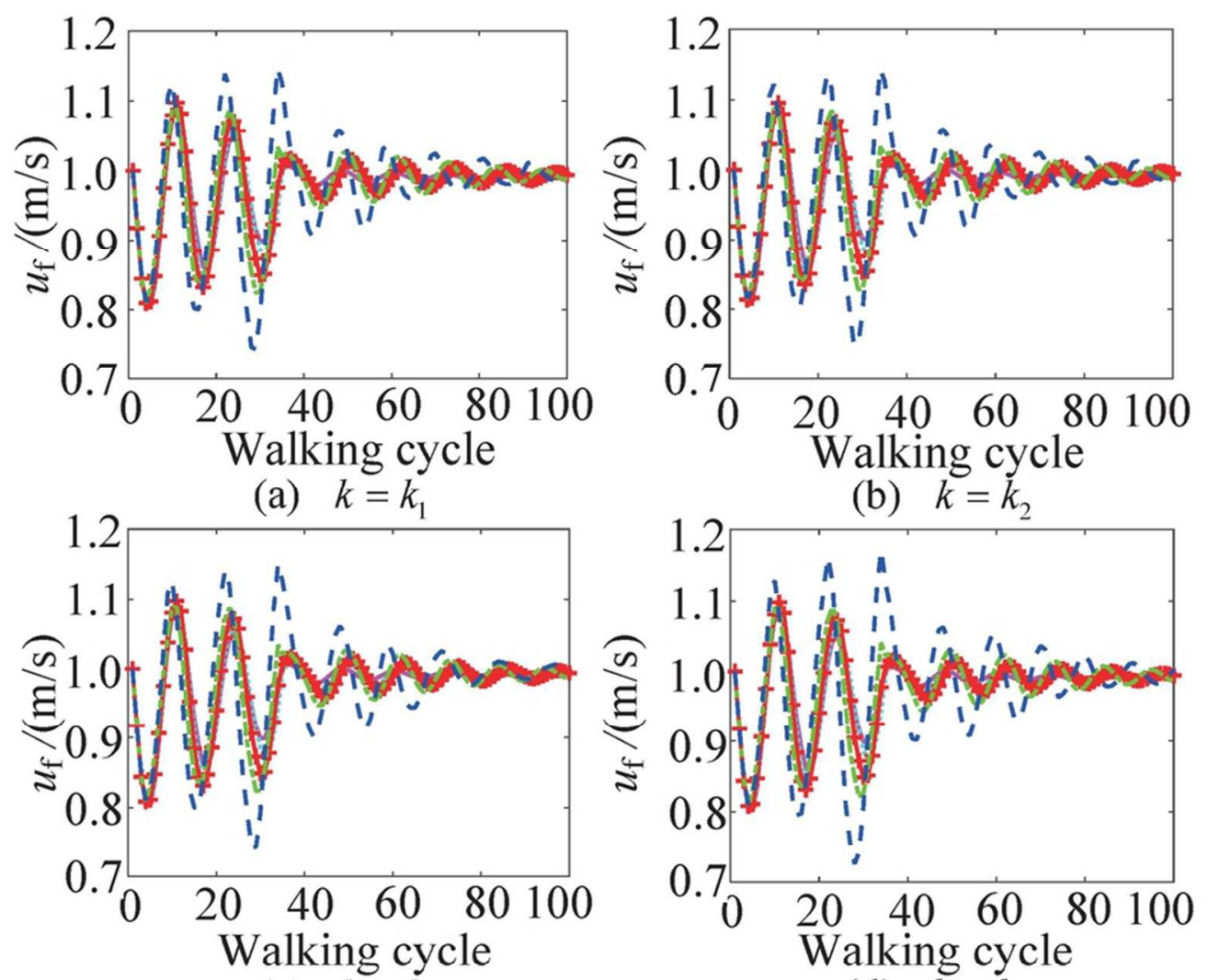

(c) $k=k_{4}$

(d) $k=k_{5}$
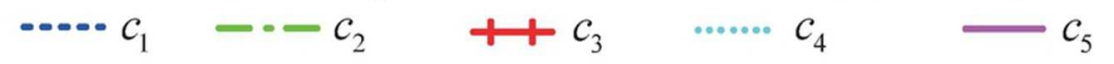

Changes in uf on a $10^{\circ}$ slope with a controller

$79 \times 71 \mathrm{~mm}(300 \times 300$ DPI $)$ 

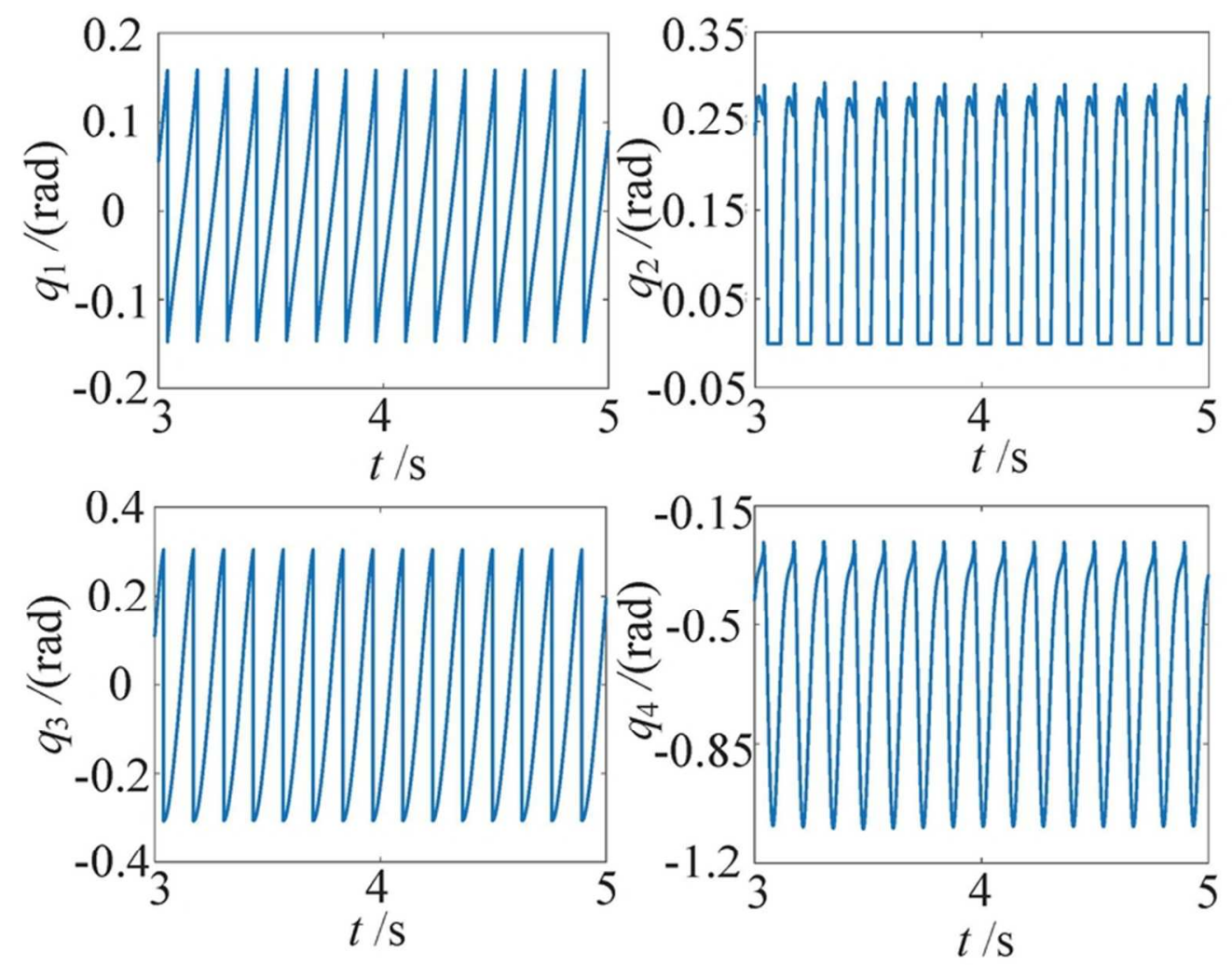

Joint angles with respect to time on a $-10^{\circ}$ slope with a controller $69 \times 54 \mathrm{~mm}(300 \times 300$ DPI $)$ 

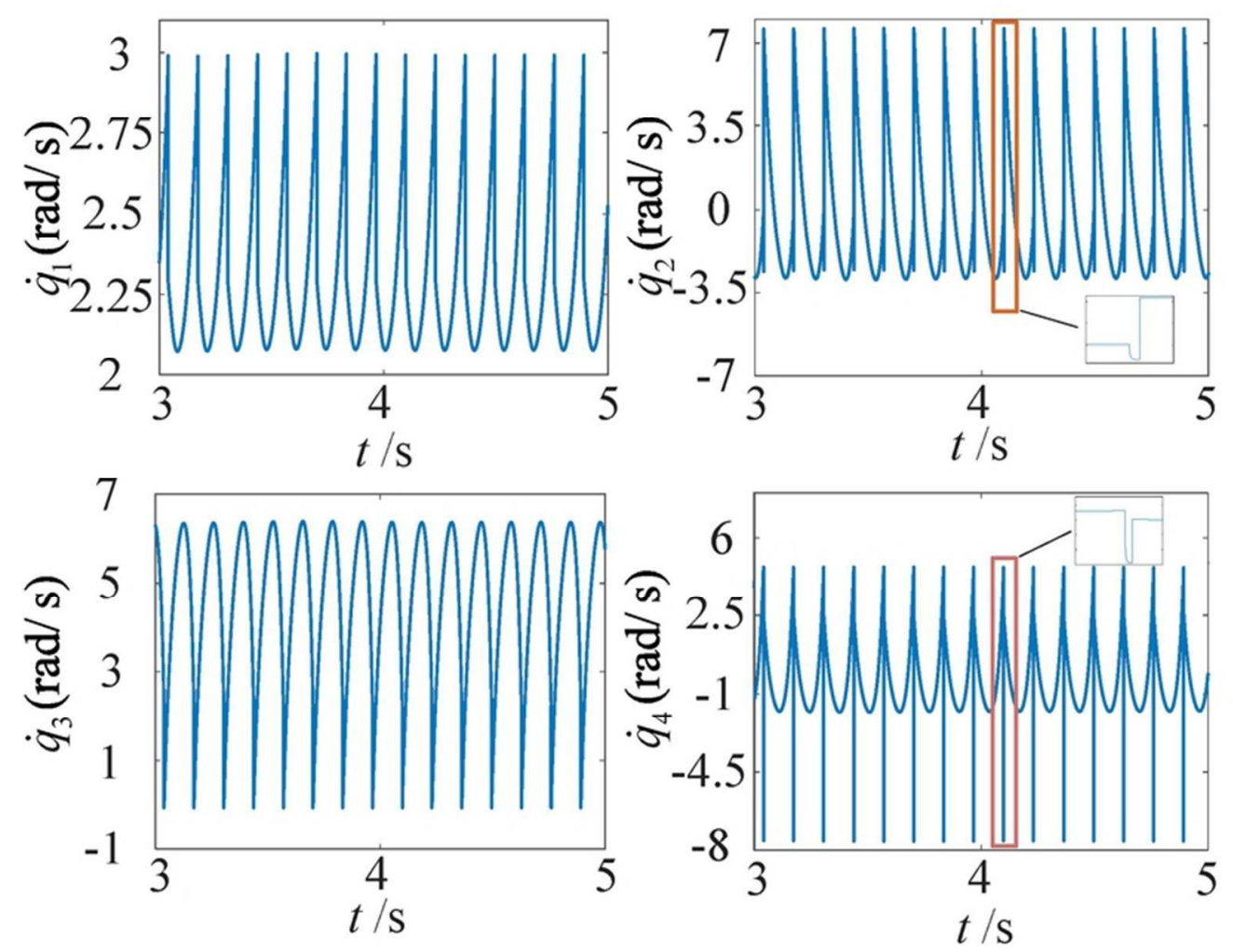

Joint velocities with respect to time on a $-10^{\circ}$ slope with a controller

$69 \times 54 \mathrm{~mm}(300 \times 300 \mathrm{DPI})$ 

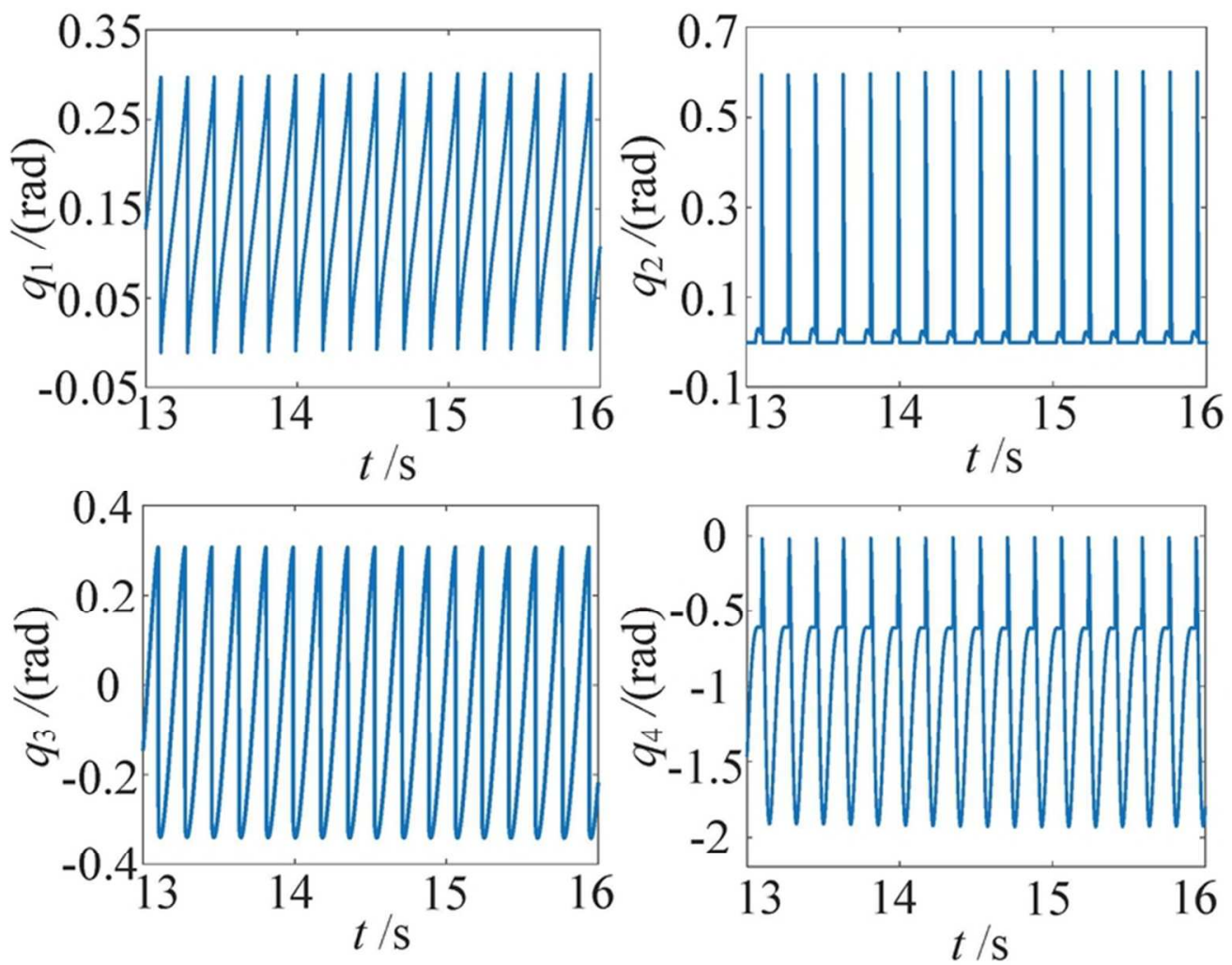

Joint angles with respect to time on a $10^{\circ}$ slope with a controller $69 \times 54 \mathrm{~mm}(300 \times 300$ DPI $)$ 


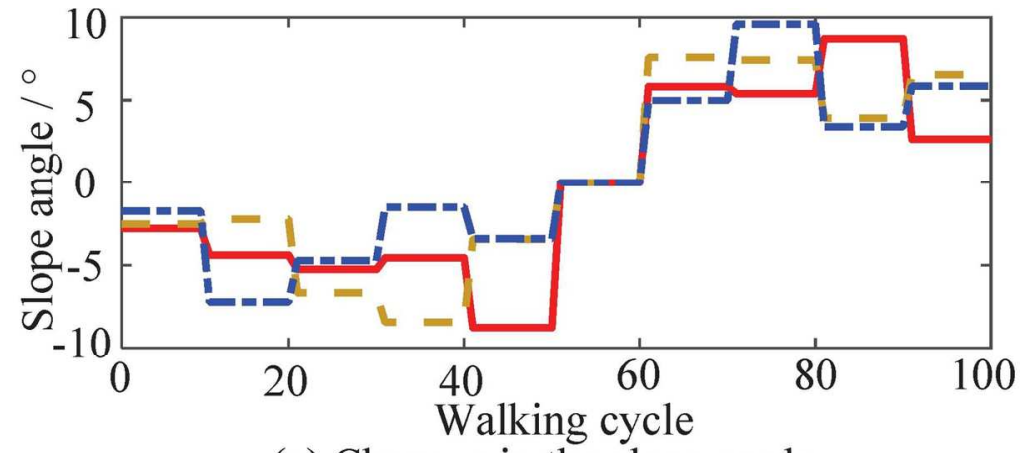

(a) Changes in the slope angle

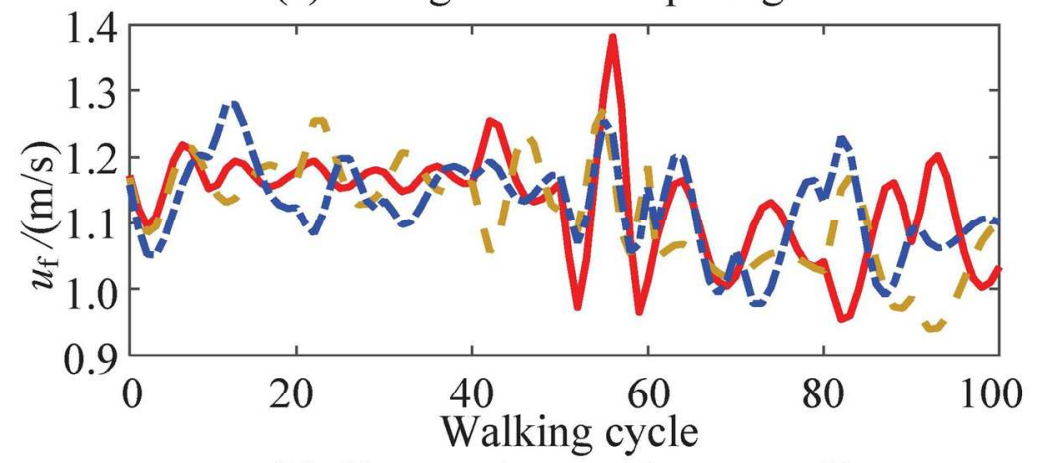

(b) Changes in $u_{\mathrm{f}}$ with a controller

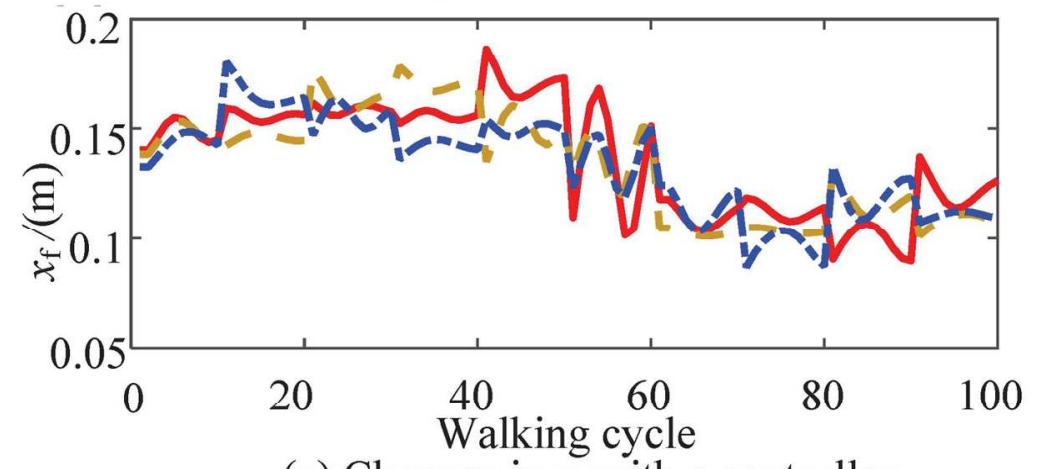

(c) Changes in $x_{\mathrm{f}}$ with a controller

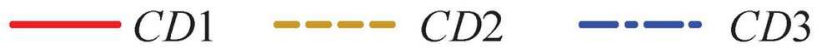

Simulation results on varying slopes with fixed compliance parameters. $122 \times 187 \mathrm{~mm}(300 \times 300 \mathrm{DPI})$ 

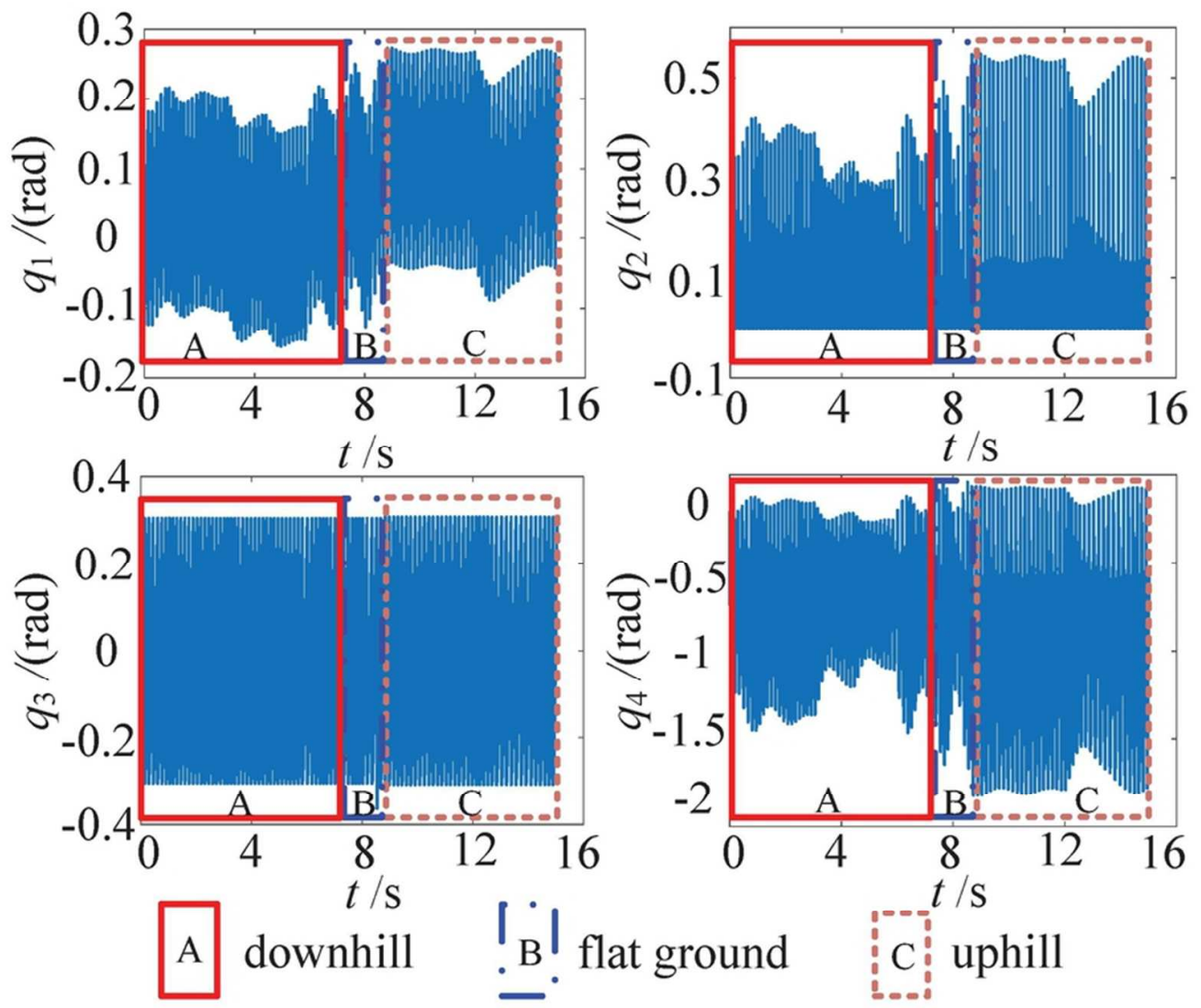

Fig. 15. Joint angles with respect to time on varying slopes with fixed compliance parameters $76 \times 66 \mathrm{~mm}(300 \times 300$ DPI $)$ 


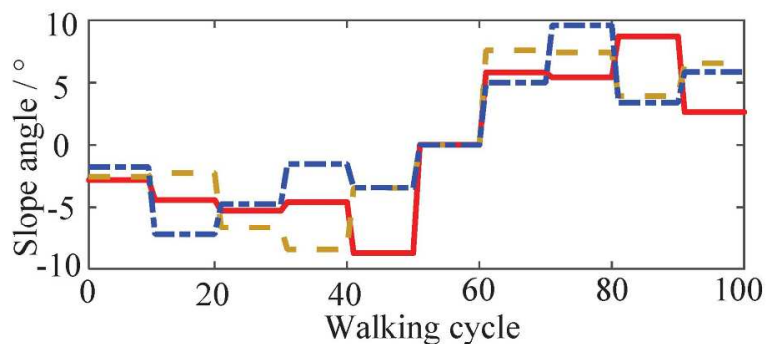

(a) Changes in the slope angle

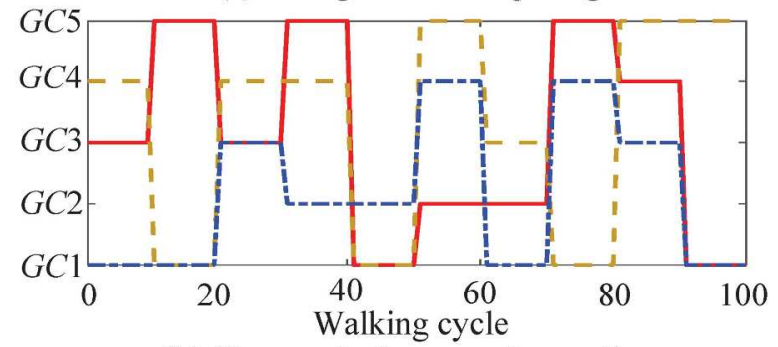

(b) Changes in the ground compliance

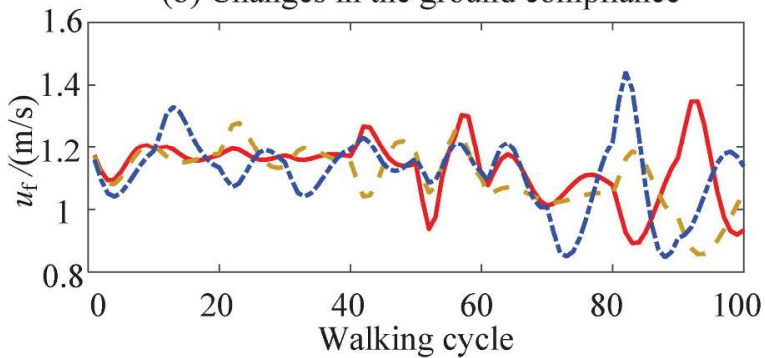

(c) Changes in $u_{\mathrm{f}}$ with a controller

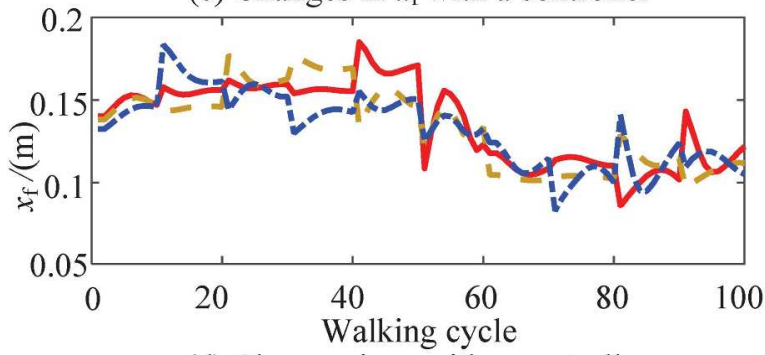

(d) Changes in $x_{\mathrm{f}}$ with a controller

$\longrightarrow C D 1$ - $--C D 2 \quad---C D 3$

Fig. 16. Simulation results on varying slopes with varying compliance parameters.

$162 \times 330 \mathrm{~mm}(300 \times 300$ DPI $)$ 

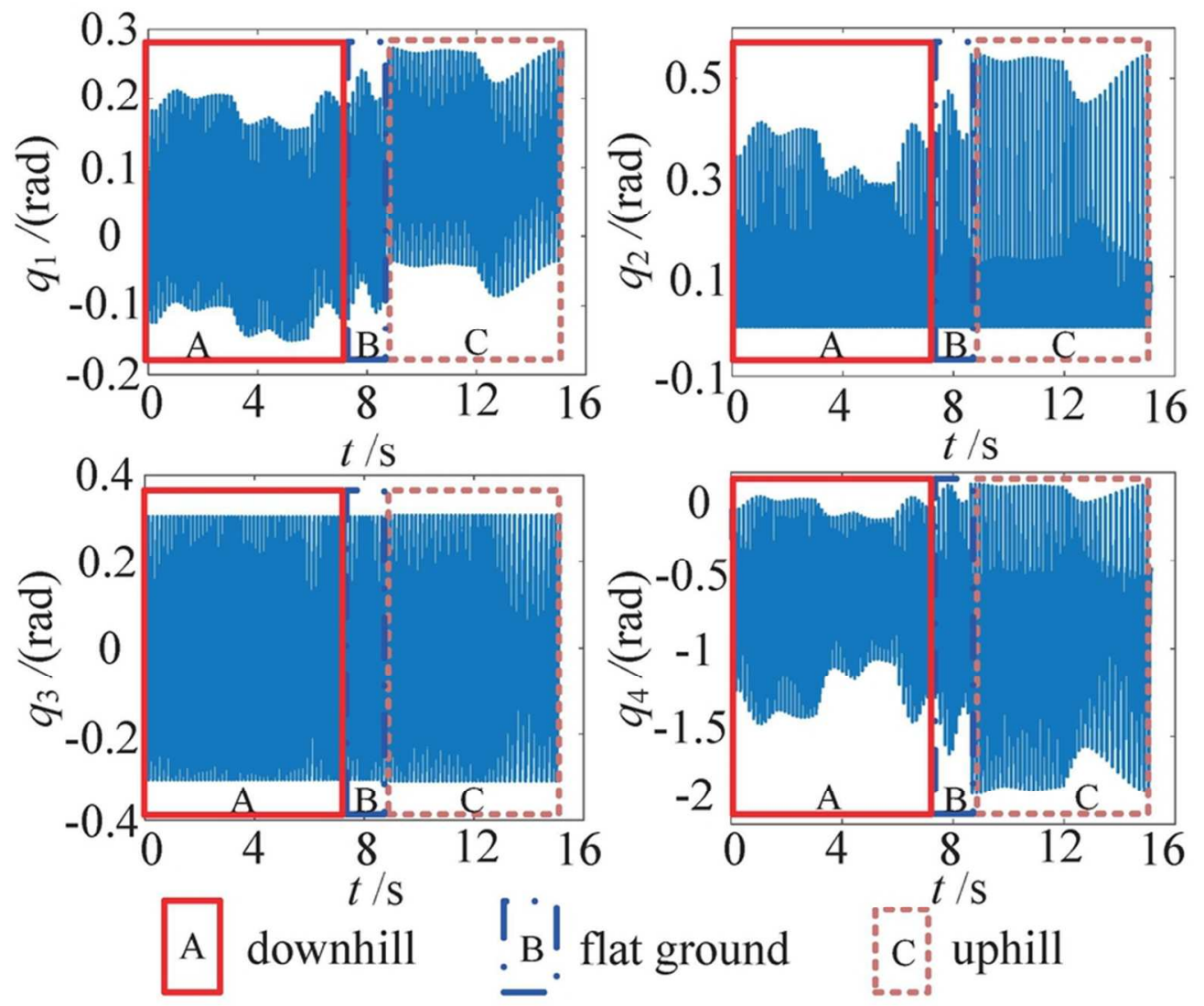

Fig. 17. Joint angles with respect to time on varying slopes with varying compliance parameters $76 \times 66 \mathrm{~mm}(300 \times 300 \mathrm{DPI})$ 


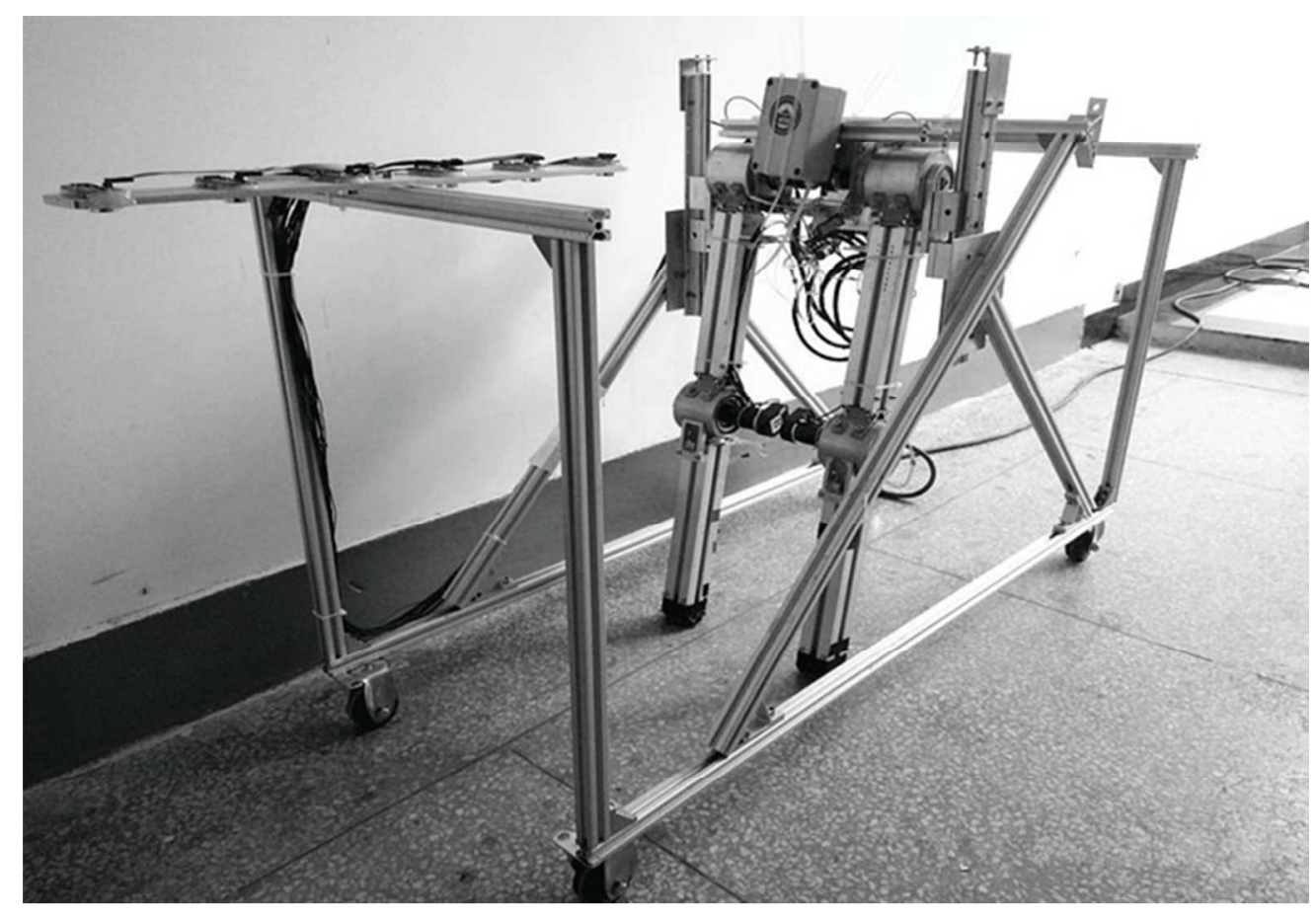

Fig. 18. Prototype robot "UBbot"

$63 \times 43 \mathrm{~mm}(300 \times 300 \mathrm{DPI})$ 


\section{Appendix}

The detailed calculation of equation (12) in this paper is as follows

$$
\begin{aligned}
u_{\mathrm{fcal}} & =U_{\mathrm{rod}}\left(\lambda, v_{\mathrm{i}}, u_{\mathrm{i}}, x_{\mathrm{i}}, x_{\mathrm{f}}, y_{\mathrm{i}}\right) \\
& =\frac{1}{4} \sqrt{\frac{3 y_{\mathrm{i}}^{2}\left(u^{2}+v^{2}+\rho^{2} \omega^{2}\right)+3(1-\lambda) y_{\mathrm{i}} g\left(x_{\mathrm{f}}^{2}-x_{\mathrm{i}}^{2}\right)}{y_{\mathrm{i}}^{2}+x_{\mathrm{f}}^{2}}}
\end{aligned}
$$

where

$$
\begin{gathered}
\rho=\sqrt{\frac{x_{\mathrm{i}}^{2}+y_{\mathrm{i}}^{2}}{3}} \\
u=u_{\mathrm{i}}-\lambda \frac{\rho^{2}}{\rho^{2}+y_{\mathrm{i}}^{2}}\left(u_{\mathrm{i}}-y_{\mathrm{i}} \omega_{\mathrm{i}}\right) \\
v=v_{\mathrm{i}}-\frac{\rho^{2} \lambda\left[2\left(v_{\mathrm{i}}+x_{\mathrm{i}} \omega_{\mathrm{i}}\right)\left(\rho^{2}+y_{\mathrm{i}}^{2}\right)+\left(u_{\mathrm{i}}-y_{\mathrm{i}} \omega_{\mathrm{i}}\right) x_{\mathrm{i}} y_{\mathrm{i}} \lambda\right]}{2\left(\rho^{2}+y_{\mathrm{i}}^{2}\right)^{2}} \\
\omega=\omega_{\mathrm{i}}-\lambda \frac{v_{\mathrm{i}} x_{\mathrm{i}}-u_{\mathrm{i}} y_{\mathrm{i}}+\omega_{\mathrm{i}}\left(x_{\mathrm{i}}^{2}+y_{\mathrm{i}}^{2}\right)}{\rho^{2}+y_{\mathrm{i}}^{2}}+\frac{\left(u_{\mathrm{i}}-y_{\mathrm{i}} \omega_{\mathrm{i}}\right) x_{\mathrm{i}}^{2} y_{\mathrm{i}} \lambda^{2}}{2\left(\rho^{2}+y_{\mathrm{i}}^{2}\right)^{2}}
\end{gathered}
$$

